\title{
Estimation of single-index model with spatial interaction
}

\author{
Yan Sun* \\ School of Economics \\ Shanghai University of Finance and Economics, P. R. China
}

\begin{abstract}
This article is concerned with the single-index model in spatial dependence data, where the spatial lag effect enters the model linearly and the relationship between variables is a nonparametric function of a linear combination of multivariate regressors. This setup avoids the so-called curse of dimensionality while still capturing important nonlinear features in high dimensional data. It also provides a convenient framework in which to model interactions between the regressors. We propose a two stage estimation strategy where the nonparametric component is established by a local linear approach and the estimation of the parametric part by GMM method, which can be seen as a direct nonlinear least squares method. We derive the asymptotic distributions of the unknowns in our model, and the procedures for constructing simultaneous confidence bands of the nonparametric function are also established. In addition, a simulation study is performed.
\end{abstract}

Keywords: spatial dependence, single-index setting, reparameterization, semiparametric GMM estimation, asymptotic normality, simultaneous confidence band.

\section{Introduction}

One objective in many real applications is to build regression models to identify the economic relationships between variables observed over a region of interest, under

*Email: sunyan@mail.shufe.edu.cn 
the assumption that the responses are spatially associated. Careful inspection of economic problems have revealed the limitations of linear regression model to explain the potential relationship between the response variable and its associated covariates. Indeed, some economic variables exhibit highly nonlinear relationships (Paelinck and Klaassen, 1979; Basile and Gress, 2005). Neglecting the underlying nonlinear relationship in spatial dependence models often leads to inconsistent estimation of the parameters of interest.

It is a distinctive challenge facing analysts of spatial econometric data to characterize certain flexible functional forms that try to account for potential nonlinearity after considering the possibility of spatial dependence. Works taking into account issues of functional form have received increasing attention in the economic literature. For example, Pace et al. (2004) and Yang et al. (2006) introduced a parametric functional form transformation on the response variable or covaraites. As a solid priori information and theoretical foundation are usually lacking in empirical studies, it is usually recommended to start with a more flexible model.

Nonparametric techniques have received many focus because of its flexibility, see Härdle (1994). Su (2012) attempts to describe the relationship between the response and all its regressors by a nonparametric function in the spatial autoregressive model and establishes its estimators. However, the nonparametric elements are subject to the curse of dimensionality (Bellman, 1961) and can only accommodate low dimensional covariates. Basile et al. (2014) focus on some recently developed spatial autoregressive semiparametric geoadditive models. The single-index model (Ichimura, 1993; Xia, 2006 ) is also one of the semiparametric framework. As it generalizes linear regression by replacing the linear combination $\boldsymbol{\delta}_{0}^{\mathrm{T}} \mathbf{x}$, where $\mathbf{x}$ is a vector of regressors, with a nonparametric function $g\left(\boldsymbol{\delta}_{0}^{\mathrm{T}} \mathbf{x}\right)$, it avoids the so-called curse of dimensionality while still capturing important nonlinear features in high dimensional data. This setup also provides a convenient framework in which to model interactions between the covariates. Further, as we only need to assume some continuity conditions of $g$ on $\boldsymbol{\delta}_{0}^{\mathrm{T}} \mathbf{x}$, it can incorporate some discrete regressors.

Estimation of single-index model using independent cross sectional data is very attractive both in theory and practice, among which the most popular ones are the 
average derivative estimation method proposed by Powell et al. (1989) and the semiparametric least square method (Ichimura, 1993; Liang et al., 2010).

This paper is concerned with the single-index model in spatial dependence data, where the spatial lag effect enters the model linearly and the relationship between variables has the form $g\left(\boldsymbol{\delta}_{0}^{\mathrm{T}} \mathbf{x}\right)$ with $g$ being a smooth function. We will propose a two stage estimation strategy for both parameters (the one dimensional spatial parameter and the multivariate single-index parameter) and the nonparametric part. First, we treat the spatial parameter and the single-index parameter as if they were known, and use the local linear method to estimate the nonparametric component. Then the generalized moment method (GMM) is used to estimate the parametric part. Given the estimates of the parameters, the final nonparametric component can be obtained. Different from various methods for estimating the single-index part (Powell et al., 1989; Ichimura 1993; Carroll et al., 1997; Xia, 2006; Liang et al., 2010), our approach can be seen as a direct least squares method by transferring the restricted least square to an unrestricted least squares which is also rapid and computationally simple using standard nonlinear least squares software.

The rest of the paper is organized as follows. Section 2 proposes the model and its estimation procedure, and Section 3 gives its asymptotic properties. In Section 4, we describe the implementation of the method and discuss the selection of the bandwidths. The simulation studies are presented in Section 5. Section 6 concludes. The technical proofs can be found in Appendix.

\section{The model and the methodology}

\subsection{The model}

Let $\left(\mathbf{x}_{n, i}, y_{n, i}\right)$ be the observation collected from the $i$ th subject $(i=1, \cdots, n)$, where $y_{n, i}$ is the response of interest, and $\mathbf{x}_{n, i} \in R^{p}$ is a $p$ dimensional regressors that do not contain constant term. We consider the following single-index model with spatial interaction:

$$
y_{n, i}=g\left(\boldsymbol{\delta}_{0}^{\mathrm{T}} \mathbf{x}_{n, i}\right)+\lambda_{0} \sum_{j \neq i} w_{n, i j} y_{n, j}+\epsilon_{n, i}, \quad i=1, \cdots, n,
$$


where $w_{n, i j}$ is a specified constant spatial weight, $g(\cdot)$ is an unknown smooth function, $\boldsymbol{\delta}_{0} \in R^{p}$ is an unknown true single-index parameter, $\lambda_{0} \in R$ is an unknown true spatial coefficient, reflecting spatial autocorrelation between neighbors, $\epsilon_{n, i}, i=1, \cdots, n$, are independent and identically distributed (iid) random errors with zero mean and finite variance $\sigma^{2}$.

The transformation function $g$ in model (2.1) is completely unspecified, it is appealing in fitting multivariate nonparametric regression functions. Here, we assume that the function $g(\cdot)$ is not constant on its support. It is also assumed that at least one nonconstant regressors is continuous and must have significant coefficients in order that valid instrument variables can be generated from them. Further, we assume that the true single-index parameter satisfies $\left\|\boldsymbol{\delta}_{0}\right\|=1$ and the first element is positive to ensure identifiability (Xia, 2006).

Basile et al. (2014) point out that spatial modelling of economic phenomena should be well equipped to simultaneously deal with spatial dependence, spatial unobserved heterogeneity and unknown functional form. They also state that spatial dependence may be the result of both spatial spillovers and spatially correlated omitted variable (known as unobserved heterogeneity). In order to properly interpret the spatial model, only spatial spillovers should be captured by the spatial lag term, while the unobserved heterogeneity can be taken into account through the inclusion of a spatial trend surface $h(l a, l o)$, i.e. a smooth interaction between latitude $(l a)$ and longitude $(l o)$. Obviously, model (2.1) can also be used to deal with these issues synchronously. When $\mathbf{x}_{n, i}$ includes both latitude and longitude, unobserved heterogeneity can be controlled and the spatial lag term can be used to assess the impact of interested externalities. Different from that of Basile et al. (2014), we use a single-index function to describe possible nonlinearities, while they use additive and varying coefficient functions. Advantages of our setup are that model interactions between covariates are automatically allowed and all the covariates are treated equal since we have little prior information at the stage of exploratory data analysis.

Another main contributions of our paper is to establish its estimation procedures and study its asymptotic properties. Basile et al. (2014) use a two-step estimation procedures to estimate spatial autoregressive geoadditive model, that is, to use penal- 
ized spline to estimate nonparametric function and control function $(\mathrm{CF})$ approach to solve endogeneity bias caused by correlation between spatial lag term and the error term. Different from theirs, we use local linear method to estimate the unknown function. Compared with the spline method, the local linear estimator is more efficient, and it is nearly optimal in an asymptotic minmax sense (Fan and Gijbels, 1996). Meanwhile, we can simultaneously obtain the estimate of the first derivative of the unknown function which is needed to calculate marginal effects. In the second step, we use instrumental variable methods GMM to deal with endogeneity rather than $\mathrm{CF}$ approach. The CF method should impose extra stricter assumptions and is hard to establish its asymptotic properties (They only give the simulation studies). It treats endogeneity as an omitted variable problem and attempts to find its proxy covariate (the part of the endogenous variable that is correlated with random error) to correct the inconsistency of least square method, while GMM use moment conditions introduced by instrument variables (the part of the endogenous variable that is uncorrelated with random error) to solve endogeniety.

\section{$2.2 \quad$ Interpretation of model (2.1)}

Because of existence of externalities in model (2.1), the interpretation of the model is quite complicated. The explanatory variable in one region will not only affect itself (direct effect) but also all the other regions (indirect effect). In the following, we will calculate these effects using the reduced form of the model.

Let $Y_{n}=\left(y_{n, 1}, \cdots, y_{n, n}\right)^{\mathrm{T}}, \boldsymbol{\epsilon}_{n}=\left(\epsilon_{n, 1}, \cdots, \epsilon_{n, n}\right)^{\mathrm{T}}, W_{n}=\left(w_{n, i j}\right)_{1 \leq i, j \leq n}, I_{n}$ be an identity matrix of size $n,\left[\left(I_{n}-\lambda_{0} W_{n}\right)^{-1}\right]_{i j}$ be the $(i, j)$ th element of $\left(I_{n}-\lambda_{0} W_{n}\right)^{-1}$, $\mathbf{g}\left(\boldsymbol{\delta}_{0}^{\mathrm{T}} X_{n}\right)=\left(g\left(\boldsymbol{\delta}_{0}^{\mathrm{T}} \mathbf{x}_{n, 1}\right), \cdots, g\left(\boldsymbol{\delta}_{0}^{\mathrm{T}} \mathbf{x}_{n, n}\right)\right)^{\mathrm{T}}, \delta_{0, l}$ and $x_{n, i l}$ be the $l$ th element of $\boldsymbol{\delta}_{0}$ and $\mathbf{x}_{n, i}$, respectively. Then the reduced form of model (2.1) can be written as

$$
y_{n, i}=\sum_{j=1}^{n}\left[\left(I_{n}-\lambda_{0} W_{n}\right)^{-1}\right]_{i j} g\left(\boldsymbol{\delta}_{0}^{\mathrm{T}} \mathbf{x}_{n, j}\right)+\sum_{j=1}^{n}\left[\left(I_{n}-\lambda_{0} W_{n}\right)^{-1}\right]_{i j} \epsilon_{n, j}, \quad i=1, \cdots, n .
$$

Without loss of generality, we assume that the $l$ th component of the explanatory variable is continuous and we get by straightforward calculations that

$$
\frac{\partial E\left(y_{n, i}\right)}{\partial x_{n, i l}}=\left[\left(I_{n}-\lambda_{0} W_{n}\right)^{-1}\right]_{i i} \delta_{0, l} g^{\prime}\left(\boldsymbol{\delta}_{0}^{\mathrm{T}} \mathbf{x}_{n, i}\right)
$$


and

$$
\frac{\partial E\left(y_{n, i}\right)}{\partial x_{n, j l}}=\left[\left(I_{n}-\lambda_{0} W_{n}\right)^{-1}\right]_{i j} \delta_{0, l} g^{\prime}\left(\boldsymbol{\delta}_{0}^{\mathrm{T}} \mathbf{x}_{n, j}\right) .
$$

Therefore, the average direct, total and indirect effects of the $l$ th explanatory variable are ( see Chap 2.7 in Lesage and Pace, 2009 )

$$
\begin{gathered}
\bar{M}(l)_{\text {direct }}=\frac{1}{n} \sum_{i=1}^{n}\left[\left(I_{n}-\lambda_{0} W_{n}\right)^{-1}\right]_{i i} \delta_{0, l} g^{\prime}\left(\boldsymbol{\delta}_{0}^{\mathrm{T}} \mathbf{x}_{n, i}\right), \\
\bar{M}(l)_{\text {total }}=\frac{1}{n} \sum_{i=1}^{n} \sum_{j=1}^{n}\left[\left(I_{n}-\lambda_{0} W_{n}\right)^{-1}\right]_{i j} \delta_{0, l} g^{\prime}\left(\boldsymbol{\delta}_{0}^{\mathrm{T}} \mathbf{x}_{n, j}\right),
\end{gathered}
$$

and

$$
\bar{M}(l)_{\text {indirect }}=\bar{M}(l)_{\text {total }}-\bar{M}(l)_{\text {direct }}
$$

respectively. For discrete variable, the effects can be obtained by substraction instead of partial derivative. Obviously, the estimates of the above effects can be obtained by replacing the parametric and nonparametric part with their estimators given in the next subsection. The bootstrap method can be used to build their confidence intervals.

\subsection{Estimation procedures}

For any given $v, g(\cdot)$ can be approximated by the Taylor expansion

$$
g(v) \approx g(u)+g^{\prime}(u)(v-u)
$$

for $v$ in a neighborhood of $u$. First we assume that $\lambda$ and $\delta$ are known, then we can find the estimator of the unknown function by minimizing

$$
\sum_{i=1}^{n}\left\{y_{n, i}^{*}-g\left(\boldsymbol{\delta}^{\mathrm{T}} \mathbf{x}\right)-g^{\prime}\left(\boldsymbol{\delta}^{\mathrm{T}} \mathbf{x}\right)\left(\boldsymbol{\delta}^{\mathrm{T}} \mathbf{x}_{n, i}-\boldsymbol{\delta}^{\mathrm{T}} \mathbf{x}\right)\right\}^{2} K_{h}\left(\boldsymbol{\delta}^{\mathrm{T}} \mathbf{x}_{n, i}-\boldsymbol{\delta}^{\mathrm{T}} \mathbf{x}\right),
$$

where $y_{n, i}^{*}=y_{n, i}-\lambda \sum_{j \neq i} w_{n, i j} y_{n, j}, K_{h}(u)=K(u / h) / h, K(\cdot)$ is a kernel function and $h$ is the bandwidth. Let $\tilde{g}\left(\boldsymbol{\delta}^{\mathrm{T}} \mathbf{x} ; \boldsymbol{\delta}, \lambda\right)$ and $\tilde{g}^{\prime}\left(\boldsymbol{\delta}^{\mathrm{T}} \mathbf{x} ; \boldsymbol{\delta}, \lambda\right)$ minimize (2.2). It follows by straightforward calculation that

$$
\left(\begin{array}{c}
\tilde{g}\left(\boldsymbol{\delta}^{\mathrm{T}} \mathbf{x} ; \boldsymbol{\delta}, \lambda\right) \\
h \tilde{g}^{\prime}\left(\boldsymbol{\delta}^{\mathrm{T}} \mathbf{x} ; \boldsymbol{\delta}, \lambda\right)
\end{array}\right)=\left\{\Psi_{n h}\left(\boldsymbol{\delta}^{\mathrm{T}} \mathbf{x} ; \boldsymbol{\delta}\right)\right\}^{-1} \mathcal{X}_{n h}^{\mathrm{T}}\left(\boldsymbol{\delta}^{\mathrm{T}} \mathbf{x} ; \boldsymbol{\delta}\right) \mathcal{K}_{n h}\left(\boldsymbol{\delta}^{\mathrm{T}} \mathbf{x} ; \boldsymbol{\delta}\right)\left(Y_{n}-\lambda W_{n} Y_{n}\right)
$$


where $\Psi_{n h}\left(\boldsymbol{\delta}^{\mathrm{T}} \mathbf{x} ; \boldsymbol{\delta}\right)=\mathcal{X}_{n h}^{\mathrm{T}}\left(\boldsymbol{\delta}^{\mathrm{T}} \mathbf{x} ; \boldsymbol{\delta}\right) \mathcal{K}_{n h}\left(\boldsymbol{\delta}^{\mathrm{T}} \mathbf{x} ; \boldsymbol{\delta}\right) \mathcal{X}_{n h}\left(\boldsymbol{\delta}^{\mathrm{T}} \mathbf{x} ; \boldsymbol{\delta}\right)$,

$$
\mathcal{X}_{n h}\left(\boldsymbol{\delta}^{\mathrm{T}} \mathbf{x} ; \boldsymbol{\delta}\right)=\left(\begin{array}{ccc}
1 & \cdots & 1 \\
\left(\boldsymbol{\delta}^{T} \mathbf{x}_{n, 1}-\boldsymbol{\delta}^{\mathrm{T}} \mathbf{x}\right) / h & \cdots & \left(\boldsymbol{\delta}^{T} \mathbf{x}_{n, n}-\boldsymbol{\delta}^{\mathrm{T}} \mathbf{x}\right) / h
\end{array}\right)^{\mathrm{T}}
$$

and

$$
\mathcal{K}_{n h}\left(\boldsymbol{\delta}^{\mathrm{T}} \mathbf{x} ; \boldsymbol{\delta}\right)=\operatorname{diag}\left(K_{h}\left(\boldsymbol{\delta}^{T} \mathbf{x}_{n, 1}-\boldsymbol{\delta}^{\mathrm{T}} \mathbf{x}\right), \cdots, K_{h}\left(\boldsymbol{\delta}^{T} \mathbf{x}_{n, n}-\boldsymbol{\delta}^{\mathrm{T}} \mathbf{x}\right)\right) .
$$

Let $Z_{n}=\left(\mathbf{z}_{n, 1}, \cdots, \mathbf{z}_{n, n}\right)^{\mathrm{T}}$ be an $n \times r(r \geq p+1)$ matrix of IVs constructed as functions of $X_{n}=\left(\mathbf{x}_{n, 1}, \cdots, \mathbf{x}_{n, n}\right)^{\mathrm{T}}$ and $W_{n}$ suggested by Kelejian and Prucha (1998), such that we have the moment functions $E\left(Z_{n}^{\mathrm{T}} \boldsymbol{\epsilon}_{n}\right)=\mathbf{0}$. For example $Z_{n}=$ $\left(\mathbf{1}, X_{n}, W_{n} X_{n}, W_{n}^{2} X_{n}\right)$, etc. Next we replace $g(\cdot)$ in model $(2.1)$ by $\tilde{g}(\cdot ; \boldsymbol{\delta}, \lambda)$ and the corresponding moment functions can be approximated by

$$
\mathbf{m}_{n}(\boldsymbol{\delta}, \lambda)=Z_{n}^{\mathrm{T}}\left[Y_{n}-\lambda W_{n} Y_{n}-\tilde{\mathbf{g}}\left(\boldsymbol{\delta}^{\mathrm{T}} X_{n} ; \boldsymbol{\delta}, \lambda\right)\right],
$$

where $\tilde{\mathbf{g}}\left(\boldsymbol{\delta}^{\mathrm{T}} X_{n} ; \boldsymbol{\delta}, \lambda\right)=\left(\tilde{g}\left(\boldsymbol{\delta}^{\mathrm{T}} \mathbf{x}_{n, 1} ; \boldsymbol{\delta}, \lambda\right), \cdots, \tilde{g}\left(\boldsymbol{\delta}^{\mathrm{T}} \mathbf{x}_{n, n} ; \boldsymbol{\delta}, \lambda\right)\right)^{\mathrm{T}}$. Let $A_{n}$ be a positive definite constant matrix of order $r$, then the GMM estimators of $\boldsymbol{\delta}$ and $\lambda$ can be found by minimizing

$$
Q_{n}(\boldsymbol{\alpha}, \lambda)=\mathbf{m}_{n}^{\mathrm{T}}(\boldsymbol{\delta}, \lambda) A_{n} \mathbf{m}_{n}(\boldsymbol{\delta}, \lambda)
$$

under the constraint $\|\boldsymbol{\delta}\|=1$ and its first component being positive. The optimal choice of $A_{n}$ for given instruments $Z_{n}$ will be discussed later.

In the following we first handle the constraint as in $\mathrm{Yu}$ and Ruppert (2002) by reparameterization. For $\boldsymbol{\delta}=\left(\delta_{1}, \cdots, \delta_{p}\right)^{\mathrm{T}}$, let $\boldsymbol{\alpha}=\left(\delta_{2}, \cdots, \delta_{p}\right)^{\mathrm{T}}$ be a $p-1$ dimensional parameter vector with the first component $\delta_{1}$ in $\boldsymbol{\delta}$ removed. Then we may write

$$
\boldsymbol{\delta}=\boldsymbol{\delta}(\boldsymbol{\alpha})=\left(\sqrt{1-\|\boldsymbol{\alpha}\|^{2}}, \boldsymbol{\alpha}^{\mathrm{T}}\right)^{\mathrm{T}} .
$$

Obviously the true parameter $\boldsymbol{\alpha}_{0}$ must satisfy the constraint $\left\|\boldsymbol{\alpha}_{0}\right\|<1$. Hence $\boldsymbol{\delta}$ is infinitely differentiable in a neighborhood of $\boldsymbol{\alpha}_{0}$. By reparameterization, the corresponding set of moment functions is

$$
\mathbf{m}_{n}(\boldsymbol{\delta}, \lambda)=\mathbf{m}_{n}(\boldsymbol{\delta}(\boldsymbol{\alpha}), \lambda)=\widetilde{\mathbf{m}}_{n}(\boldsymbol{\alpha}, \lambda)
$$

and the objective GMM function is

$$
Q_{n}(\boldsymbol{\delta}, \lambda)=Q_{n}(\boldsymbol{\delta}(\boldsymbol{\alpha}), \lambda)=\widetilde{Q}_{n}(\boldsymbol{\alpha}, \lambda) .
$$


Therefore, the GMM estimator of the finite dimensional parameter $\left(\boldsymbol{\alpha}^{\mathrm{T}}, \lambda\right)^{\mathrm{T}}$ is

$$
\left(\hat{\boldsymbol{\alpha}}^{\mathrm{T}}, \hat{\lambda}\right)^{\mathrm{T}}=\operatorname{argmin}_{(\boldsymbol{\alpha}, \lambda)} \widetilde{Q}_{n}(\boldsymbol{\alpha}, \lambda)=\operatorname{argmin}_{(\boldsymbol{\alpha}, \lambda)} \widetilde{\mathbf{m}}_{n}^{\mathrm{T}}(\boldsymbol{\alpha}, \lambda) A_{n} \widetilde{\mathbf{m}}_{n}(\boldsymbol{\alpha}, \lambda),
$$

and then the estimator of $\boldsymbol{\delta}$ is $\hat{\boldsymbol{\delta}}=\boldsymbol{\delta}(\hat{\boldsymbol{\alpha}})$.

The final estimator $\hat{g}(\cdot)$ of $g(\cdot)$ is taken as $\tilde{g}(\cdot ; \boldsymbol{\delta}, \lambda)$ with $\boldsymbol{\delta}, \lambda$ being replaced by $\hat{\boldsymbol{\delta}}, \hat{\lambda}$, and the bandwidth $h$ by a slightly larger bandwidth $h_{1}$.

The above proposed methods estimate nonparametric functions and parameters by minimizing two different loss functions (2.2) and (2.4), respectively. As is well known, the bias of the estimation of $g(\cdot)$ using $(2.2)$ exists when the optimal bandwidth is used. This will lead to a slower convergence rate for the estimators of parameters than root-n. Therefore, undersmoothing the nonparametric function at the first stage is needed to eliminate the bias and allow the estimators of parameters to achieve root-n consistency, see Carroll et al.(1997) and Wang et al.(2010), among others. Hence, to obtain the optimal convergence rates of estimations of parametric and nonparametric part respectively, the bandwidths required are different, where $h$ is used to calculate $\hat{\boldsymbol{\delta}}$ and $\hat{\lambda}$, while $h_{1}$ is used to calculate $\hat{g}(\cdot)$.

\section{$3 \quad$ Asymptotic properties}

In this section, we will present the asymptotic properties for the established estimators of the linear single-index model with spatial interaction. To clear exposition, we first introduce the following notations. Let $\hat{\boldsymbol{\xi}} \equiv\left(\hat{\boldsymbol{\delta}}^{\mathrm{T}}, \hat{\lambda}\right)^{\mathrm{T}}, \boldsymbol{\xi}_{0} \equiv\left(\boldsymbol{\delta}_{0}^{\mathrm{T}}, \lambda_{0}\right)^{\mathrm{T}}, M_{n} \equiv$

$$
\begin{aligned}
& W_{n}\left(I_{n}-\lambda_{0} W_{n}\right)^{-1}, J\left(\boldsymbol{\alpha}_{0}\right) \equiv\left(\begin{array}{c}
-\frac{\boldsymbol{\alpha}_{0}^{T}}{\sqrt{1-\left\|\boldsymbol{\alpha}_{0}\right\|^{2}}} \\
I_{p-1}
\end{array}\right), \quad J_{1} \equiv \operatorname{diag}\left(J\left(\boldsymbol{\alpha}_{0}\right), 1\right), \\
& \mathbf{s}_{n h}\left(\boldsymbol{\delta}_{0}^{\mathrm{T}} \mathbf{x} ; \boldsymbol{\delta}_{0}\right) \equiv(1,0)\left\{\Psi_{n h}\left(\boldsymbol{\delta}_{0}^{\mathrm{T}} \mathbf{x} ; \boldsymbol{\delta}_{0}\right)\right\}^{-1} \mathcal{X}_{n h}^{\mathrm{T}}\left(\boldsymbol{\delta}_{0}^{\mathrm{T}} \mathbf{x} ; \boldsymbol{\delta}_{0}\right) \mathcal{K}_{n h}\left(\boldsymbol{\delta}_{0}^{\mathrm{T}} \mathbf{x} ; \boldsymbol{\delta}_{0}\right), \quad i=1, \cdots, n, \\
& S_{n h}\left(\boldsymbol{\delta}_{0}^{\mathrm{T}} X_{n} ; \boldsymbol{\delta}_{0}\right) \equiv\left(\mathbf{s}_{n h}^{\mathrm{T}}\left(\boldsymbol{\delta}_{0}^{\mathrm{T}} \mathbf{x}_{n, 1} ; \boldsymbol{\delta}_{0}\right), \cdots, \mathbf{s}_{n h}^{\mathrm{T}}\left(\boldsymbol{\delta}_{0}^{\mathrm{T}} \mathbf{x}_{n, n} ; \boldsymbol{\delta}_{0}\right)\right)^{\mathrm{T}} \\
& \Gamma_{1, n} \equiv \frac{1}{n} \sum_{i=1}^{n} g^{\prime}\left(\boldsymbol{\delta}_{0}^{\mathrm{T}} \mathbf{x}_{n, i}\right) \mathbf{z}_{n, i} \mathbf{x}_{n, i}^{\mathrm{T}}, \quad \Gamma_{2, n} \equiv \frac{1}{n} Z_{n}^{\mathrm{T}}\left[I_{n}-S_{n h}\left(\boldsymbol{\delta}_{0}^{\mathrm{T}} X_{n} ; \boldsymbol{\delta}_{0}\right)\right] M_{n} \mathbf{g}\left(\boldsymbol{\delta}_{0}^{\mathrm{T}} X_{n}\right),
\end{aligned}
$$

and

$$
\Sigma_{n} \equiv \frac{1}{n} Z_{n}^{\mathrm{T}}\left[I_{n}-S_{n h}\left(\boldsymbol{\delta}_{0}^{\mathrm{T}} X_{n} ; \boldsymbol{\delta}_{0}\right)\right]\left[I_{n}-S_{n h}\left(\boldsymbol{\delta}_{0}^{\mathrm{T}} X_{n} ; \boldsymbol{\delta}_{0}\right)\right]^{\mathrm{T}} Z_{n} .
$$

We first impose the following assumptions. They are possibly not the weakest ones, but they are convenient for technical proofs. 


\section{Conditions}

(1) The kernel function $K(\cdot)$ is a continuous symmetric density function with a compact support on R. In addition, its first derivative is bounded and continuous.

(2) $\mathbf{x}_{n, i}, i=1, \cdots, n$ are uniformly bounded constant on $\mathcal{X}$; and there exists a positive density function $f(\cdot)$ such that

$$
\frac{1}{n} \sum_{i=1}^{n} r\left(t_{i}\right) K_{h}\left(t_{i}-t\right)-\int r(s) K_{h}(s-t) f(s) d s=o(1)
$$

uniformly in $t \in \mathcal{R}$ for any bounded continuous function $r(\cdot)$, and $K(\cdot)$ satisfies Condition (1). $f(\cdot)$ is bounded away from zero and satisfies a Lipshitz condition on a compact support $\mathcal{R}$, where $\mathcal{R}=\left\{t=\boldsymbol{\delta}^{\mathrm{T}} \mathbf{x}: \boldsymbol{\delta} \in \Delta, \mathbf{x} \in \mathcal{X}\right\}$ with $\Delta$ and $\mathcal{X}$ being compact supports.

(3) The elements $\mathbf{z}_{n, i}$ of $Z_{n}$ are uniformly bounded constants.

(4) The function $g(\cdot)$ is twice continuously differentiable and not constant on the support $\mathcal{R}$. Further, $g(\cdot), g^{\prime}(\cdot)$ and $g^{\prime \prime}(\cdot)$ are all bounded on its support.

(5) As a normalization, $w_{n, i i}=0$ for all $i$. The matrix $I_{n}-\lambda W_{n}$ is nonsingular for all $\lambda$ in its compact support. Furthermore, the matrices $W_{n}$ and $\left(I_{n}-\lambda_{0} W_{n}\right)^{-1}$ are uniformly bounded in both row and column sums in absolute value.

(6) $\epsilon_{n, i}$, are iid with zero mean and finite variance $\sigma^{2}$. Moreover, $E\left|\epsilon_{n, i}\right|^{q}<\infty$ for some $q>2$.

(7) The true parameter $\left(\boldsymbol{\delta}_{0}^{\mathrm{T}}, \lambda_{0}\right)^{\mathrm{T}}$ is an interior point in its compact support.

(8) $\lim _{n \rightarrow \infty} A_{n}=A$ where $A$ is positive definite. Moreover, $\Gamma_{1}=\lim _{n \rightarrow \infty} \Gamma_{1, n}, \Gamma_{2}=$ $\lim _{n \rightarrow \infty} \Gamma_{2, n}$ and $\Sigma=\lim _{n \rightarrow \infty} \Sigma_{n}$ exist, where $\Sigma$ is positive definite.

(9) $n \rightarrow \infty, h \rightarrow 0, n h^{2} \rightarrow \infty, n h^{4} \rightarrow 0, h_{1} \rightarrow 0$, and $n h_{1}^{5}=O(1)$.

Remark 1: The fixed bounded design in Conditions (2)-(3) is typically assumed in spatial econometric literature, see Kelejian and Prucha (1998, 1999), Lee (2004) and 
Su and Jin (2010) for example. As in Linton (1995), Condition (2) does not preclude $\left\{\mathbf{x}_{n, i}\right\}_{i=1}^{n}$ from being generated by some random mechanism. For example, if $\mathbf{x}_{n, i}$ were iid and $f(\cdot)$ were the density function of $\boldsymbol{\delta}^{T} \mathbf{x}_{n, i}$, then Condition (2) holds with probability one, which follows immediately from Mack and Silverman (1982). In this case, we can obtain our results by first conditional on $\left\{\mathbf{x}_{n, i}, \mathbf{z}_{n, i}\right\}_{i=1}^{n}$ and then go on as usual.

Remark 2: Condition (5) concerns the essential features of the weight matrix for the model. This condition is originated in the work of Kelejian and Prucha (1998). Lee (2007) and $\mathrm{Su}(2012)$ also assume Condition (5) to limit the correlation between spatial units in a manageable degree. In fact, the matrices are uniformly bounded in row or column sums in absolute value is equivalent to the boundedness of the row or column sums matrix norm, respectively (Horn and Johnson, 1985). Hence, it is obvious by the property of matrix norm that the product of two matrices $C_{n}$ and $D_{n}$ which are both uniformly bounded in row or column sums in absolute value are uniformly bounded in row or column sums in absolute value, respectively.

Now we are going to present the asymptotic results of the proposed estimators when $n \rightarrow \infty$, and the technical proofs can be found in Appendix.

Theorem 1. Under Conditions (1)-(8), $h \rightarrow 0, n h^{2} \rightarrow \infty$, and $n h^{4} \rightarrow 0$, the GMM estimator $\hat{\boldsymbol{\xi}}$ derived from minimizing $Q_{n}(\boldsymbol{\delta}, \lambda)$ under constraint $\|\boldsymbol{\delta}\|=1$ and its first component being positive, has the asymptotic distribution

$$
\sqrt{n}\left(\hat{\boldsymbol{\xi}}-\boldsymbol{\xi}_{0}\right) \stackrel{D}{\rightarrow} \mathbf{N}\left(\mathbf{0}, \sigma^{2} J_{1}\left(\Gamma^{\mathrm{T}} A \Gamma\right)^{-1} \Gamma^{\mathrm{T}} A \Sigma A^{\mathrm{T}} \Gamma\left(\Gamma^{\mathrm{T}} A \Gamma\right)^{-1} J_{1}^{\mathrm{T}}\right)
$$

under the assumption that $\Gamma^{\mathrm{T}} A \Gamma$ has full rank, and $\Gamma \equiv\left(\Gamma_{1} J\left(\boldsymbol{\alpha}_{0}\right), \Gamma_{2}\right)$.

It can be seen from Theorem 1 that the optimal choice of the weight matrix $A_{n}$ is $\Sigma_{n}^{-1}$ by generalized Schwartz inequality. The estimator $\hat{\Sigma}_{n}$ of $\Sigma_{n}$ can be obtained with $\boldsymbol{\delta}_{0}$ replaced by $\hat{\boldsymbol{\delta}}$. The following theorem shows that the feasible optimal GMM estimator with $\hat{\Sigma}_{n}$ has the same limiting distribution as that of the optimal GMM estimator based on $\Sigma_{n}$.

Theorem 2. Under the conditions of Theorem 1, we have that $\hat{\Sigma}_{n}-\Sigma_{n}=o_{P}(1)$, and the feasible optimal GMM estimator $\hat{\boldsymbol{\xi}}_{\text {op }}$ based on (2.4) with $A_{n}$ replaced by $\hat{\Sigma}_{n}^{-1}$ 
has the asymptotic distribution

$$
\sqrt{n}\left(\hat{\boldsymbol{\xi}}_{o p}-\boldsymbol{\xi}_{0}\right) \stackrel{D}{\rightarrow} \mathbf{N}\left(\mathbf{0}, \sigma^{2} J_{1}\left(\Gamma^{\mathrm{T}} \Sigma^{-1} \Gamma\right)^{-1} J_{1}^{\mathrm{T}}\right)
$$

Theorem 3. Under Conditions (1)-(9), then for any given $\mathbf{x} \in \mathcal{X}$, we have

$$
\sqrt{n h_{1}}\left\{\hat{g}\left(\hat{\boldsymbol{\delta}}^{\mathrm{T}} \mathbf{x} ; \hat{\boldsymbol{\delta}}, \hat{\lambda}\right)-g\left(\boldsymbol{\delta}_{0}^{\mathrm{T}} \mathbf{x}\right)-\frac{1}{2} \mu_{2} h_{1}^{2} g^{\prime \prime}\left(\boldsymbol{\delta}_{0}^{\mathrm{T}} \mathbf{x}\right)\right\} \stackrel{D}{\rightarrow} N\left(0, \sigma^{2} \nu_{0} / f\left(\boldsymbol{\delta}_{0}^{\mathrm{T}} \mathbf{x}\right)\right),
$$

where $\nu_{0}=\int K^{2}(v) d v$.

Theorem 3 shows $\hat{g}\left(\hat{\boldsymbol{\delta}}^{\mathrm{T}} \mathbf{x} ; \hat{\boldsymbol{\delta}}, \hat{\lambda}\right)$ has the asymptotic properties as that of the local linear estimator with $\boldsymbol{\delta}_{0}$ being known, and achieves rate of $n^{-2 / 5}$, which is the optimal rate for nonparametric estimation. It means that we can select the optimal bandwidth $h_{1}$ by generalized cross validation when the sample size becomes large. It is obvious that there is a bias in the estimator of $g(\cdot)$ when the optimal bandwidth is used.

Remark: An important inference question is whether the nonparametric regression function $g(\cdot)$ in model $(2.1)$ takes some parametric form such as linear function, so that one can assess if the parametric setting in real applications is reasonable or not. This can be easily answered by the simultaneous confidence band. To to do, we first have to find ways to get consistent estimators of $g^{\prime \prime}(\cdot)$ and $f(\cdot)$ appeared in Theorem 3. For example, we can use kernel estimation. Another way to get their consistent estimators is to use the estimators we obtained in Section 2. From the proof of Theorem 3 , it can be seen that

$$
\begin{aligned}
\operatorname{bias}\left\{\hat{g}\left(\hat{\boldsymbol{\delta}}^{\mathrm{T}} \mathbf{x} ; \hat{\boldsymbol{\delta}}, \hat{\lambda}\right)\right\} & =\mathbf{s}_{n h_{1}}\left(\boldsymbol{\delta}_{0}^{\mathrm{T}} \mathbf{x} ; \boldsymbol{\delta}_{0}\right) \mathbf{g}\left(\boldsymbol{\delta}_{0}^{\mathrm{T}} X_{n}\right)-g\left(\boldsymbol{\delta}_{0}^{\mathrm{T}} \mathbf{x}\right)+o\left(h_{1}^{2}\right) \\
& =\frac{1}{2} \mu_{2} h_{1}^{2} g^{\prime \prime}\left(\boldsymbol{\delta}_{0}^{\mathrm{T}} \mathbf{x}\right)+o\left(h_{1}^{2}\right),
\end{aligned}
$$

and

$$
\begin{aligned}
\operatorname{var}\left\{\hat{g}\left(\hat{\boldsymbol{\delta}}^{\mathrm{T}} \mathbf{x} ; \hat{\boldsymbol{\delta}}, \hat{\lambda}\right)\right\} & =\sigma^{2} \mathbf{s}_{n h_{1}}\left(\boldsymbol{\delta}_{0}^{\mathrm{T}} \mathbf{x} ; \boldsymbol{\delta}_{0}\right) \mathbf{s}_{n h_{1}}^{\mathrm{T}}\left(\boldsymbol{\delta}_{0}^{\mathrm{T}} \mathbf{x} ; \boldsymbol{\delta}_{0}\right)+o\left(\left\{n h_{1}\right\}^{-1}\right) \\
& =\frac{\sigma^{2} \nu_{0}}{n h_{1} f\left(\boldsymbol{\delta}_{0}^{\mathrm{T}} \mathbf{x}\right)}+o\left(\left\{n h_{1}\right\}^{-1}\right)
\end{aligned}
$$

uniformly in $\boldsymbol{\delta}_{0}^{\mathrm{T}} \mathbf{x} \in \mathcal{R}$. Therefore, the consistent estimators $\widehat{\operatorname{bias}}(\cdot)$ and $\widehat{\operatorname{var}}(\cdot)$ can be easily obtained as

$$
\widehat{\operatorname{bias}}\left\{\hat{g}\left(\hat{\boldsymbol{\delta}}^{\mathrm{T}} \mathbf{x} ; \hat{\boldsymbol{\delta}}, \hat{\lambda}\right)\right\}=\mathbf{s}_{n h_{1}}\left(\hat{\boldsymbol{\delta}}^{\mathrm{T}} \mathbf{x} ; \hat{\boldsymbol{\delta}}\right) \hat{\mathbf{g}}\left(\hat{\boldsymbol{\delta}}^{\mathrm{T}} X_{n} ; \hat{\boldsymbol{\delta}}, \hat{\lambda}\right)-\hat{g}\left(\hat{\boldsymbol{\delta}}^{\mathrm{T}} \mathbf{x} ; \hat{\boldsymbol{\delta}}, \hat{\lambda}\right)
$$


and

$$
\widehat{\operatorname{var}}\left\{\hat{g}\left(\hat{\boldsymbol{\delta}}^{\mathrm{T}} \mathbf{x} ; \hat{\boldsymbol{\delta}}, \hat{\lambda}\right)\right\}=\hat{\sigma}^{2} \mathbf{s}_{n h_{1}}\left(\hat{\boldsymbol{\delta}}^{\mathrm{T}} \mathbf{x} ; \hat{\boldsymbol{\delta}}\right) \mathbf{s}_{n h_{1}}^{\mathrm{T}}\left(\hat{\boldsymbol{\delta}}^{\mathrm{T}} \mathbf{x} ; \hat{\boldsymbol{\delta}}\right)
$$

where $\hat{\sigma}^{2}$ is estimated by the residual sums of square. This, together with Theorem 3 provides pointwise confidence intervals for $g(\cdot)$, which can be used together with Bonferroni's inequality to obtain simultaneous confidence intervals. These $(1-\alpha)$ bands take the form

$$
\hat{g}\left(\hat{\boldsymbol{\delta}}^{\mathrm{T}} \mathbf{x}_{n, i} ; \hat{\boldsymbol{\delta}}, \hat{\lambda}\right)-\widehat{\operatorname{bias}}\left\{\hat{g}\left(\hat{\boldsymbol{\delta}}^{\mathrm{T}} \mathbf{x}_{n, i} ; \hat{\boldsymbol{\delta}}, \hat{\lambda}\right)\right\} \pm z_{\frac{\alpha}{2 n}}\left[\widehat{\operatorname{var}}\left\{\hat{g}\left(\hat{\boldsymbol{\delta}}^{\mathrm{T}} \mathbf{x}_{n, i} ; \hat{\boldsymbol{\delta}}, \hat{\lambda}\right)\right\}\right]^{1 / 2}, \quad i=1, \cdots, n
$$

with $z_{\frac{\alpha}{2 n}}$ being the $100\left(1-\frac{\alpha}{2 n}\right)$ th percentile of the standard normal distribution.

Obviously, the above Bonferroni-type band for $g(\cdot)$ are rather conservative, just as one referee points out. Then the next theorem gives the distribution of the maximum discrepancy between the estimated function and its true value. It can be directly and easily used to construct the simultaneous confidence band with asymptotic confidence level $1-\alpha$, see Theorem 1 and its remark in Fan and Zhang (2000). To do so, we need to assume the following stronger version of Condition (2)

$$
\frac{1}{n} \sum_{i=1}^{n} r\left(t_{i}\right) K_{h}\left(t_{i}-t\right)-\int r(s) K_{h}(s-t) f(s) d s=o(1 / \log n)
$$

uniformly in $t \in \mathcal{R}$. If $t_{i}$ were iid random variables, then (3.3) is true by Mack and Silverman (1982).

Theorem 4. Under the conditions of Theorem 1, with (3.3) being true, $K(t)$ twice continuously on its compact support $\left[-c_{0}, c_{0}\right]$ and $h_{1}=n^{-\eta}, 1 / 5 \leq \eta<1 / 3$, then for all $\boldsymbol{\delta}_{0}^{\mathrm{T}} \mathbf{x} \in[a, b]$, we have

$$
\begin{aligned}
& P\left(( - 2 \operatorname { l o g } \{ h _ { 1 } / ( b - a ) \} ) ^ { 1 / 2 } \operatorname { s u p } _ { \boldsymbol { \delta } _ { 0 } ^ { \mathrm { T } } \mathbf { x } \in [ a , b ] } \left\{\mid \frac{\hat{g}\left(\hat{\boldsymbol{\delta}}^{\mathrm{T}} \mathbf{x} ; \hat{\boldsymbol{\delta}}, \hat{\lambda}\right)-g\left(\boldsymbol{\delta}_{0}^{\mathrm{T}} \mathbf{x}\right)-\operatorname{bias}\left\{\hat{g}\left(\hat{\boldsymbol{\delta}}^{\mathrm{T}} \mathbf{x} ; \hat{\boldsymbol{\delta}}, \hat{\lambda}\right)\right\}}{\left[\operatorname{var}\left\{\hat{g}\left(\hat{\boldsymbol{\delta}}^{\mathrm{T}} \mathbf{x} ; \hat{\boldsymbol{\delta}}, \hat{\lambda}\right)\right\}\right]^{1 / 2} \mid}\right.\right. \\
& \left.\left.\quad-l_{n}\right\} \leq x\right) \\
& \rightarrow \exp \{-2 \exp \{-x\}\},
\end{aligned}
$$

where

$l_{n}=\left(-2 \log \left\{h_{1} /(b-a)\right\}\right)^{1 / 2}+\frac{1}{\left(-2 \log \left\{h_{1} /(b-a)\right\}\right)^{1 / 2}}\left[\log \frac{K^{2}\left(c_{0}\right)}{\nu_{0} \pi^{1 / 2}}+\frac{1}{2} \log \log \left\{(b-a) / h_{1}\right\}\right]$ 
if $K\left(c_{0}\right) \neq 0$ and

$$
l_{n}=\left(-2 \log \left\{h_{1} /(b-a)\right\}\right)^{1 / 2}+\frac{1}{\left(-2 \log \left\{h_{1} /(b-a)\right\}\right)^{1 / 2}} \log \left\{\frac{1}{4 \nu_{0} \pi} \int\left(K^{\prime}(t)\right)^{2} d t\right\}
$$

if $K\left(c_{0}\right)=0$.

Remark: Based on Theorem 4 and consistent estimators of $\operatorname{bias}(\cdot)$ and $\operatorname{var}(\cdot)$, the asymptotic $1-\alpha$ confidence band of $g(\cdot)$ on $[a, b]$ can be easily constructed as

$$
\hat{g}\left(\hat{\boldsymbol{\delta}}^{\mathrm{T}} \mathbf{x} ; \hat{\boldsymbol{\delta}}, \hat{\lambda}\right)-\widehat{\operatorname{bias}}\left\{\hat{g}\left(\hat{\boldsymbol{\delta}}^{\mathrm{T}} \mathbf{x} ; \hat{\boldsymbol{\delta}}, \hat{\lambda}\right)\right\} \pm \gamma_{\alpha}\left[\widehat{\operatorname{var}}\left\{\hat{g}\left(\hat{\boldsymbol{\delta}}^{\mathrm{T}} \mathbf{x} ; \hat{\boldsymbol{\delta}}, \hat{\lambda}\right)\right\}\right]^{1 / 2}
$$

where

$$
\gamma_{\alpha}=l_{n}+[\log 2-\log \{-\log (1-\alpha)\}]\left(-2 \log \left\{h_{1} /(b-a)\right\}\right)^{-1 / 2}
$$

\section{Implementation}

\subsection{Algorithm}

In the following, we will specify a simple algorithm for calculating the estimators.

Step 0: Select an initial estimator $\hat{\boldsymbol{\xi}}=\left(\hat{\boldsymbol{\delta}}^{\mathrm{T}}, \hat{\lambda}\right)^{\mathrm{T}}$. For example, let $\lambda=0$ and then fit a parametric linear model $y=\boldsymbol{\delta}^{\mathrm{T}} \mathbf{x}+\epsilon$ by ordinary least squares to get $\hat{\boldsymbol{\zeta}}$. Set $\hat{\boldsymbol{\delta}}=\operatorname{sign}\left(\hat{\zeta}_{1}\right) \cdot \hat{\boldsymbol{\zeta}} /\|\hat{\boldsymbol{\zeta}}\|$ to satisfy the identification condition, where $\operatorname{sign}\left(\hat{\boldsymbol{\zeta}}_{1}\right)$ is the $\operatorname{sign}$ of the first element of $\hat{\zeta}$. Alternatively, an initial estimate can also be obtained by the GMM estimator of $Y_{n}=X_{n} \boldsymbol{\delta}+\lambda W_{n} Y_{n}+\epsilon_{n}$.

Step 1: With the estimated $\hat{\boldsymbol{\delta}}$, find the estimates of the index value $\left\{v_{n, i}=\right.$ $\left.\hat{\boldsymbol{\delta}}^{\mathrm{T}} \mathbf{x}_{n, i} ; i=1, \cdots, n\right\}$, then obtain $\tilde{g}(v ; \hat{\boldsymbol{\delta}}, \hat{\lambda})$ by $(2.3)$. Here the choice of bandwidth $h$ will be given in the following subsection.

Step 2: With the estimated $\tilde{g}(v ; \hat{\boldsymbol{\delta}}, \hat{\lambda})$, update $\hat{\boldsymbol{\delta}}$ and $\hat{\lambda}$ by minimizing $Q_{n}(\boldsymbol{\delta}, \lambda)$ with respect to all the components of $(\boldsymbol{\delta}, \lambda)$ under the constraint that $\|\boldsymbol{\delta}\|=1$ and its first element is larger than zero to realize the minimization problem.

Step 3: Continue step 1 and 2 until convergence.

Step 4: Given $(\boldsymbol{\delta}, \lambda)$ at its final estimate from Step 3, the final estimate of $g(\cdot)$ is taken as $\tilde{g}(v ; \boldsymbol{\delta}, \lambda)$ with the bandwidth $h_{1}$ to be the bandwidth that is optimal for estimation of $g(\cdot)$, which will be given in the following subsection. 
Remark: Step 2 can be realized by using standard nonlinear least square (NLS) algorithms. In this article, we will adopt NLS function lsqnonlin() from Matlab's optimization toolbox in the following simulation studies.

\subsection{Bandwidth selection}

The optimal bandwidth used in the final step $\hat{h}_{1, \text { opt }}$ is selected by generalized cross validation $(\mathrm{GCV})$, that is

$$
\hat{h}_{1, \text { opt }}=\operatorname{argmin}_{h_{1}} G C V\left(h_{1}\right)
$$

where

$$
G C V\left(h_{1}\right)=\frac{\frac{1}{n} \bar{Y}_{n}^{\mathrm{T}}\left(I_{n}-S_{n h_{1}}\left(\hat{\boldsymbol{\delta}}^{\mathrm{T}} X_{n} ; \hat{\boldsymbol{\delta}}\right)\right)^{\mathrm{T}}\left(I_{n}-S_{n h_{1}}\left(\hat{\boldsymbol{\delta}}^{\mathrm{T}} X_{n} ; \hat{\boldsymbol{\delta}}\right)\right) \bar{Y}_{n}}{\left[1-\frac{1}{n} \operatorname{tr}\left(S_{n h_{1}}\left(\hat{\boldsymbol{\delta}}^{\mathrm{T}} X_{n} ; \hat{\boldsymbol{\delta}}\right)\right)\right]^{2}}
$$

and $\bar{Y}_{n}=Y_{n}-\hat{\lambda} W_{n} Y_{n}$.

A sensible rule for choice of $h$ in step 1 is more difficult to obtain. A relatively ad hoc possibility (Carroll et al., 1997) is

$$
\hat{h}=\hat{h}_{1, o p t} \cdot n^{1 / 5} \cdot n^{-1 / 3}=\hat{h}_{1, o p t} \cdot n^{-2 / 15}
$$

because it guarantees that the bandwidth to derive the estimation of constant parameters has the correct order of magnitude.

\section{Simulation study}

In this section we will conduct a Monte Carlo simulation to study the finite sample performance of the proposed estimates. Like Su (2012), the spatial weight matrix $W_{n}$ is generated according to Rook continuity, by randomly distributing the $n$ spatial units on a lattice of $5 \times m(\geq n)$ squares, finding the neighbors for each unit, and then row normalizing.

Consider the following data generated processes:

$$
y_{n, i}=\sin \left(\left(\boldsymbol{\delta}_{0}^{\mathrm{T}} \mathbf{x}_{n, i}-A\right) \pi\right)+B+\lambda_{0} \sum_{j \neq i} w_{n, i j} y_{n, j}+\epsilon_{n, i}, \quad i=1, \cdots, n,
$$


where $A=0.4, B=0.3, \boldsymbol{\delta}_{0}^{\mathrm{T}}=(1,1,1) / \sqrt{3}, \lambda_{0}=0.3, \mathbf{x}_{n, i}=\left(x_{n 1, i}, x_{n 2, i}, x_{n 3, i}\right)^{\mathrm{T}}$ with each component $x_{n j, i}, j=1,2,3$, being independently generated from $U[0,1]$ and $\epsilon_{n, i}$ independently from $N\left(0,0.2^{2}\right)$.

For the local linear estimator of $g(\cdot)$, we use Epanechikov kernel $K(t)=0.75(1-$ $\left.t^{2}\right)_{+}$as the kernel function. The GMM estimation considered here are a simple unweighted GMM approach (GMM) and an feasible optimal GMM approach (OGMM) using $Z_{n}=\left(\mathbf{1}, X_{n}, W_{n} X_{n}, W_{n}^{2} X_{n}\right)$, respectively.

For each given sample size, we do 500 repetitions. The results are reported in Table 1, where the sample bias, standard error and root mean squared error are listed to assess the accuracy of the estimates of the unknown constant parameters. Also the sample root mean average square error (RMASE) defined as

$$
\operatorname{RMASE}=\sqrt{\frac{1}{n} \sum_{i=1}^{n}\left\{\hat{g}\left(\hat{\boldsymbol{\delta}}^{\mathrm{T}} \mathbf{x}_{n, i} ; \hat{\boldsymbol{\delta}}, \hat{\lambda}\right)-g\left(\boldsymbol{\delta}_{0}^{\mathrm{T}} \mathbf{x}_{n, i}\right)\right\}^{2}}
$$

is also listed to assess the estimator of the unknown function, see Table 2. Meanwhile, we single out the estimated unknown function with median performance among the 500 simulations, see Figure 1.

It is obvious that the SE and RMSE of the OGMM estimates are smaller than those of the GMMs, and the estimates behave better when the sample size becomes large.

\section{Conclusion}

In this article, we propose a single-index model with spatial interaction to capture certain possible nonlinear shapes between the response and the regressors besides spatial dependence between neighbors. Such setting avoids the curse of dimensionality in fitting multivariate nonparametric functions. Then we propose a two stage semiparametric estimation strategy and develop the asymptotic theories for the estimators. Although the error terms in our model are assumed to be homoscedastic, the estimators can be easily extended to the heteroscedastic errors.

In the beginning, we point out that nonparametric setting is flexible and is helpful for preliminary data analysis. Simultaneous confidence bands obtained from Theorem 
Table 1: Simulation results for the parameter estimates

\begin{tabular}{|c|c|c|c|c|c|c|c|c|}
\hline \multirow{2}{*}{$\begin{array}{c}\text { Parameter } \\
n=100\end{array}$} & \multicolumn{3}{|c|}{ GMM } & \multicolumn{5}{|c|}{ OGMM } \\
\hline & Mean & Bias & $\mathrm{SE}$ & RMSE & Mean & Bias & $\mathrm{SE}$ & RMSE \\
\hline$\delta_{1}$ & 0.5707 & -0.0067 & 0.1053 & 0.1054 & 0.5719 & -0.0055 & 0.0987 & 0.0990 \\
\hline$\delta_{2}$ & 0.5694 & -0.0079 & 0.1063 & 0.1068 & 0.5690 & -0.0084 & 0.0949 & 0.0954 \\
\hline$\delta_{3}$ & 0.5614 & -0.0160 & 0.1121 & 0.1131 & 0.5668 & -0.0106 & 0.0964 & 0.0970 \\
\hline$\lambda_{0}$ & 0.3131 & 0.0131 & 0.3930 & 0.3932 & 0.3151 & 0.0151 & 0.2652 & 0.2657 \\
\hline \multicolumn{9}{|l|}{$\mathrm{n}=400$} \\
\hline$\delta_{1}$ & 0.5718 & -0.0056 & 0.0675 & 0.0678 & 0.5753 & -0.0020 & 0.0512 & 0.0510 \\
\hline$\delta_{2}$ & 0.5743 & -0.0031 & 0.0752 & 0.0755 & 0.5753 & -0.0020 & 0.0559 & 0.0557 \\
\hline$\delta_{3}$ & 0.5725 & -0.0048 & 0.0733 & 0.0735 & 0.5738 & -0.0036 & 0.0551 & 0.0557 \\
\hline$\lambda_{0}$ & 0.3237 & 0.0337 & 0.2584 & 0.2606 & 0.3106 & 0.0206 & 0.1983 & 0.1992 \\
\hline
\end{tabular}

NOTE: Shown are the sample mean (Mean), standard error (SE), bias (Bias) and root mean squared error (RMSE) of the parameter estimates from 500 simulation.

Table 2: Simulation results for the nonparameter estimate

\begin{tabular}{ccccc}
\hline \hline \multirow{2}{*}{ function } & & with GMM & & with OGMM \\
$g(\cdot)$ & $\mathrm{n}=100$ & 0.5499 & & 0.3771 \\
& $\mathrm{n}=400$ & 0.3610 & & 0.2771 \\
\hline \hline
\end{tabular}

3 and 4 can shed some light on the form of the unknown function $g(\cdot)$. Inference on testing the functional form of $g(\cdot)$, especially whether $g(\cdot)$ is a linear function, is important for empirical analysis, which can be formally tested by Theorem 4. Just as one referee points out that we can have more efficient GMM estimator constructed from both $Z_{n}$ and quadratic moments, see Lee (2007). 

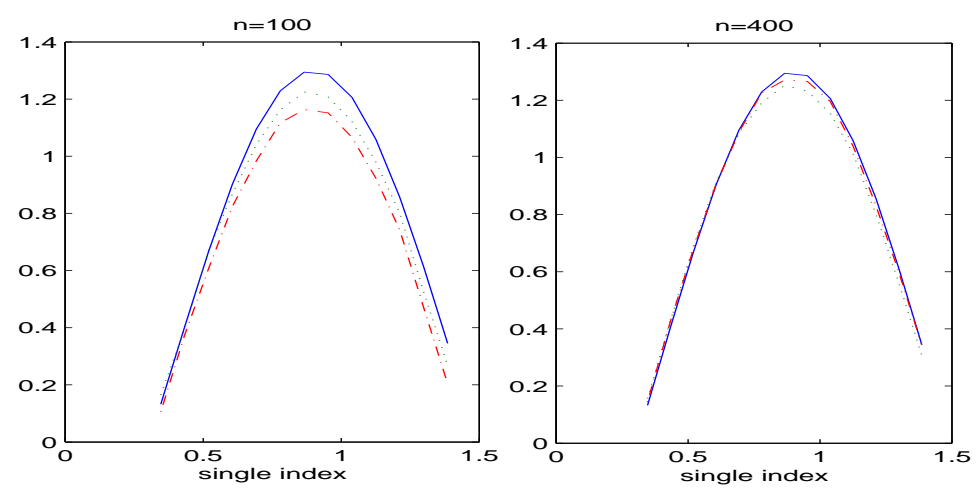

Figure 1: Curve estimate for a single replication of $g(\cdot)$. The solid curve is the true function, the dotted and dash-dotted curve are respectively the median performance fit over 500 simulation under GMM and OGMM parameter estimates.

\section{Appendix: Proof of the theorems}

First, we introduce some notations for clear exposition. Throughout the paper, we let $C$ to denote a positive constant which may take different values at each appearance and $I(D)$ be a function which takes one if $D$ is true, otherwise takes zero. $\|\Upsilon\|$ denotes Frobenius norm when $\Upsilon$ is a matrix and $\|\Upsilon\|=\left(\Upsilon^{\mathrm{T}} \Upsilon\right)^{1 / 2}$ when $\Upsilon$ is a vector. Further, let $\mathcal{T}=\{(\boldsymbol{\delta}, \mathbf{x}): \boldsymbol{\delta} \in \Delta, \mathbf{x} \in \mathcal{X}\}, \mathcal{T}_{n}=\left\{(\boldsymbol{\delta}, \mathbf{x}):(\boldsymbol{\delta}, \mathbf{x}) \in \mathcal{T},\left\|\boldsymbol{\delta}-\boldsymbol{\delta}_{0}\right\| \leq C n^{-1 / 2}\right\}$ for some positive constant $C>0, \mu_{j}=\int v^{j} K(v) d v, \nu_{j}=\int v^{j} K^{2}(v) d v, j=0,1,2$, and $\mathbf{s}_{n h}^{*}\left(\boldsymbol{\delta}^{\mathrm{T}} \mathbf{x} ; \boldsymbol{\delta}\right)=(0,1)\left\{\Psi_{n h}\left(\boldsymbol{\delta}^{\mathrm{T}} \mathbf{x} ; \boldsymbol{\delta}\right)\right\}^{-1} \mathcal{X}_{n h}^{\mathrm{T}}\left(\boldsymbol{\delta}^{\mathrm{T}} \mathbf{x} ; \boldsymbol{\delta}\right) \mathcal{K}_{n h}\left(\boldsymbol{\delta}^{\mathrm{T}} \mathbf{x} ; \boldsymbol{\delta}\right)$.

Next we state four lemmas, their detailed proofs can be found from the long version of the paper which is available upon request.

Lemma 1. Under Conditions (1), (2), (6), and $n h / \log n \rightarrow \infty$, we have

$$
\sup _{(\boldsymbol{\delta}, \mathbf{x}) \in \mathcal{T}}\left|\frac{1}{n} \sum_{i=1}^{n} K_{h}\left(\boldsymbol{\delta}^{\mathrm{T}} \mathbf{x}_{n, i}-\boldsymbol{\delta}^{\mathrm{T}} \mathbf{x}\right) \epsilon_{n, i}\right|=O_{P}\left(\left\{\frac{\log n}{n h}\right\}^{1 / 2}\right) .
$$

Proof: It can be shown by the same lines as the proof of Lemma 1 of Sun et al. (2014). That is, first we truncate $\epsilon_{n, i}$ and deal with the truncation error $\epsilon_{n, i} I\left(\left|\epsilon_{n, i}\right|>\right.$ $\left.\tau_{n}\right)$ by Markov inequality, where $\tau_{n}=\gamma_{n}^{-1 /(q-1)}$ and $\gamma_{n}=\{n h / \log n\}^{-1 / 2}$. Second, for the truncated process $\epsilon_{n, i} I\left(\left|\epsilon_{n, i}\right| \leq \tau_{n}\right)$, we replace the supremum with a maximization over finite intervals, and then use Bernstain inequality. 
Lemma 2. Under Conditions (1)-(8), and $h \rightarrow 0$, then we have that

(1) $n^{-1} \Psi_{n h}\left(\boldsymbol{\delta}^{\mathrm{T}} \mathbf{x} ; \boldsymbol{\delta}\right)=f\left(\boldsymbol{\delta}^{\mathrm{T}} \mathbf{x}\right)\left(\begin{array}{cc}1 & 0 \\ 0 & \mu_{2}\end{array}\right)+o(1)$ holds uniformly in $(\boldsymbol{\delta}, \mathbf{x}) \in \mathcal{T}$;

(2) $\left\{\Psi_{n h}\left(\boldsymbol{\delta}^{\mathrm{T}} \mathbf{x} ; \boldsymbol{\delta}\right)\right\}^{-1} \mathcal{X}_{n h}^{\mathrm{T}}\left(\boldsymbol{\delta}^{\mathrm{T}} \mathbf{x} ; \boldsymbol{\delta}\right) \mathcal{K}_{n h}\left(\boldsymbol{\delta}^{\mathrm{T}} \mathbf{x} ; \boldsymbol{\delta}\right) \mathbf{g}\left(\boldsymbol{\delta}^{\mathrm{T}} X_{n}\right)-\left(g\left(\boldsymbol{\delta}^{\mathrm{T}} \mathbf{x}\right), h g^{\prime}\left(\boldsymbol{\delta}^{\mathrm{T}} \mathbf{x}\right)\right)^{\mathrm{T}}=$ $\left(\begin{array}{c}\frac{1}{2} \mu_{2} h^{2} g^{\prime \prime}\left(\boldsymbol{\delta}^{\mathrm{T}} \mathbf{x}\right) \\ 0\end{array}\right)+o\left(h^{2}\right)$ holds uniformly in $(\boldsymbol{\delta}, \mathbf{x}) \in \mathcal{T}$

(3) $\sup _{(\boldsymbol{\delta}, \mathbf{x}) \in \mathcal{T}}\left|\mathbf{s}_{n h}\left(\boldsymbol{\delta}^{\mathrm{T}} \mathbf{x} ; \boldsymbol{\delta}\right) W_{n} Y_{n}\right|=O_{P}(1) \quad$ and $\sup _{(\boldsymbol{\delta}, \mathbf{x}) \in \mathcal{T}}\left|\mathbf{s}_{n h}^{*}\left(\boldsymbol{\delta}^{\mathrm{T}} \mathbf{x} ; \boldsymbol{\delta}\right) W_{n} Y_{n}\right|=O_{P}(1) ;$

(4) $\sup _{(\boldsymbol{\delta}, \mathbf{X}) \in \mathcal{T}_{n}}\left|\mathbf{s}_{n h}\left(\boldsymbol{\delta}^{\mathrm{T}} \mathbf{x} ; \boldsymbol{\delta}\right) \mathbf{g}\left(\boldsymbol{\delta}_{0}^{\mathrm{T}} X_{n}\right)-g\left(\boldsymbol{\delta}_{0}^{\mathrm{T}} \mathbf{x}\right)\right|=O\left(h^{2}+n^{-1 / 2}\right) ;$

(5) $\sup _{(\boldsymbol{\delta}, \mathbf{x}) \in \mathcal{T}_{n}}\left|\tilde{g}^{\prime}\left(\boldsymbol{\delta}^{\mathrm{T}} \mathbf{x} ; \boldsymbol{\delta}, \lambda_{0}\right)-g^{\prime}\left(\boldsymbol{\delta}_{0}^{\mathrm{T}} \mathbf{x}\right)-h^{-1} \mathbf{s}_{n h}^{*}\left(\boldsymbol{\delta}^{\mathrm{T}} \mathbf{x} ; \boldsymbol{\delta}\right) \boldsymbol{\epsilon}_{n}\right|=O_{P}\left(\left\{n h^{2}\right\}^{-1 / 2}+h\right)$.

Proof: These results can be obtained by Condition (2) and straightforward but tedious calculation.

Lemma 3. Under Conditions (1)-(8), $h \rightarrow 0$ and $n h^{2} \rightarrow \infty$, we have that (1) $\frac{1}{n} Z_{n}^{\mathrm{T}}\left[S_{n h}\left(\boldsymbol{\delta}_{0}^{\mathrm{T}} X_{n} ; \boldsymbol{\delta}_{0}\right)-S_{n h}\left(\boldsymbol{\delta}^{\mathrm{T}} X_{n} ; \boldsymbol{\delta}\right)\right] M_{n} \mathbf{g}\left(\boldsymbol{\delta}_{0}^{\mathrm{T}} X_{n}\right)=o(1)$ when $\left\|\boldsymbol{\delta}_{0}-\boldsymbol{\delta}\right\| \leq C n^{-1 / 2}$; (2) $\frac{1}{n} Z_{n}^{\mathrm{T}}\left[I_{n}-S_{n h}\left(\boldsymbol{\delta}_{0}^{\mathrm{T}} X_{n} ; \boldsymbol{\delta}_{0}\right)\right] \mathbf{g}\left(\boldsymbol{\delta}_{0}^{\mathrm{T}} X_{n}\right)=O\left(h^{2}\right)$.

Proof: These results can be obtained by Condition (2), Lemma 2(1) and straightforward but tedious calculation.

Lemma 4. Under Conditions (1)-(8), and $h \rightarrow 0$, we have that $\frac{1}{n} Z_{n}^{\mathrm{T}}\left[I_{n}-\right.$ $\left.S_{n h}\left(\boldsymbol{\delta}^{\mathrm{T}} X_{n} ; \boldsymbol{\delta}\right)\right] M_{n} \boldsymbol{\epsilon}_{n}=o_{P}(1), \frac{1}{\sqrt{n}} Z_{n}^{\mathrm{T}}\left[I_{n}-S_{n h}\left(\boldsymbol{\delta}_{0}^{\mathrm{T}} X_{n} ; \boldsymbol{\delta}_{0}\right)\right] \boldsymbol{\epsilon}_{n}=O_{P}(1)$, and $\frac{1}{\sqrt{n}} Z_{n}^{\mathrm{T}}\left[I_{n}-\right.$ $\left.S_{n h}\left(\boldsymbol{\delta}_{0}^{\mathrm{T}} X_{n} ; \boldsymbol{\delta}_{0}\right)\right] \boldsymbol{\epsilon}_{n} \stackrel{D}{\rightarrow} \mathbf{N}\left(\mathbf{0}, \sigma^{2} \Sigma\right)$.

Proof: It can be shown by Lemma 2(1) and Lemma A.4 of Lee (2007).

Proof of Theorem 1: Let $\boldsymbol{\theta}=\left(\boldsymbol{\alpha}^{\mathrm{T}}, \lambda\right)^{\mathrm{T}}, \boldsymbol{\theta}_{0}=\left(\boldsymbol{\alpha}_{0}^{\mathrm{T}}, \lambda_{0}\right)^{\mathrm{T}}$, and $\mathcal{B}_{n}=\{\boldsymbol{\theta}$ : $\left.\left\|\boldsymbol{\theta}-\boldsymbol{\theta}_{0}\right\|=B n^{-1 / 2}\right\}$ for some constant $B>0$. When $\boldsymbol{\theta} \in \mathcal{B}_{n}$, it is obvious that $\left\|\boldsymbol{\alpha}-\boldsymbol{\alpha}_{0}\right\| \leq\left\|\boldsymbol{\theta}-\boldsymbol{\theta}_{0}\right\|=B n^{-1 / 2}$, which implies that $\left\|\boldsymbol{\delta}-\boldsymbol{\delta}_{0}\right\| \leq B_{1} n^{-1 / 2}$ for some $B_{1}>0$. The proofs are as follows. Since

$$
\begin{aligned}
\sqrt{1-\|\boldsymbol{\alpha}\|^{2}}-\sqrt{1-\left\|\boldsymbol{\alpha}_{0}\right\|^{2}} & =-\frac{2 \boldsymbol{\alpha}_{0}^{\mathrm{T}}\left(\boldsymbol{\alpha}-\boldsymbol{\alpha}_{0}\right)+\left\|\boldsymbol{\alpha}-\boldsymbol{\alpha}_{0}\right\|^{2}}{\sqrt{1-\|\boldsymbol{\alpha}\|^{2}}+\sqrt{1-\left\|\boldsymbol{\alpha}_{0}\right\|^{2}}} \\
& =-\frac{\boldsymbol{\alpha}_{0}^{\mathrm{T}}\left(\boldsymbol{\alpha}-\boldsymbol{\alpha}_{0}\right)}{\sqrt{1-\left\|\boldsymbol{\alpha}_{0}\right\|^{2}}}+o\left(n^{-1 / 2}\right),
\end{aligned}
$$


we obtain that $\left\|\boldsymbol{\delta}-\boldsymbol{\delta}_{0}\right\| \leq B_{1} n^{-1 / 2}$.

In the following, we will firstly show that there is a $\operatorname{root} \hat{\boldsymbol{\theta}}$ of $\frac{\partial \widetilde{Q}_{n}(\boldsymbol{\theta})}{\partial \boldsymbol{\theta}}=0$ satisfying $\left\|\hat{\boldsymbol{\theta}}-\boldsymbol{\theta}_{0}\right\|=O_{P}\left(n^{-1 / 2}\right)$. Using result 6.3.4 of Ortega and Rheinboldt (1973), it suffices to show that $\left(\boldsymbol{\theta}-\boldsymbol{\theta}_{0}\right)^{\mathrm{T}} \frac{\partial \widetilde{Q}_{n}(\boldsymbol{\theta})}{\partial \boldsymbol{\theta}}>0$ for all $\boldsymbol{\theta} \in \mathcal{B}_{n}$ in probability. In the remaining of the proof, we assume $\boldsymbol{\theta} \in \mathcal{B}_{n}$.

It is obvious by Taylor expansion of $\widetilde{\mathbf{m}}(\boldsymbol{\theta})$ at its true value $\boldsymbol{\theta}_{0}=\left(\boldsymbol{\alpha}_{0}^{\mathrm{T}}, \lambda_{0}\right)^{\mathrm{T}}$ that

$$
\frac{\partial \widetilde{Q}_{n}(\boldsymbol{\theta})}{\partial \boldsymbol{\theta}}=2\left[\frac{\partial \widetilde{\mathbf{m}}_{n}(\boldsymbol{\theta})}{\partial \boldsymbol{\theta}^{\mathrm{T}}}\right]^{\mathrm{T}} A_{n}\left\{\widetilde{\mathbf{m}}_{n}\left(\boldsymbol{\theta}_{0}\right)+\frac{\partial \widetilde{\mathbf{m}}_{n}(\overline{\boldsymbol{\theta}})}{\partial \boldsymbol{\theta}^{\mathrm{T}}}\left(\boldsymbol{\theta}-\boldsymbol{\theta}_{0}\right)\right\}
$$

where $\overline{\boldsymbol{\theta}}$ lies between $\boldsymbol{\theta}$ and $\boldsymbol{\theta}_{0}$, hence $\left\|\overline{\boldsymbol{\theta}}-\boldsymbol{\theta}_{0}\right\| \leq B n^{-1 / 2}$.

Now we have by reparameterization that

$$
\frac{\partial \widetilde{\mathbf{m}}_{n}(\boldsymbol{\theta})}{\partial \boldsymbol{\theta}^{\mathrm{T}}}=\left(\frac{\partial \mathbf{m}_{n}(\boldsymbol{\delta}, \lambda)}{\partial \boldsymbol{\delta}^{T}} J(\boldsymbol{\alpha}), \frac{\partial \mathbf{m}_{n}(\boldsymbol{\theta})}{\partial \lambda}\right) .
$$

Then it follows by straightforward calculation, Lemma 2(3), 2(5) and Chebyshev inequality that

$$
\begin{aligned}
-\frac{1}{n} \frac{\partial \mathbf{m}_{n}(\boldsymbol{\delta}, \lambda)}{\partial \boldsymbol{\delta}^{T}}= & \frac{1}{n} \sum_{i=1}^{n}\left[\tilde{g}^{\prime}\left(\boldsymbol{\delta}^{\mathrm{T}} \mathbf{x}_{n, i} ; \boldsymbol{\delta}, \lambda\right)-\tilde{g}^{\prime}\left(\boldsymbol{\delta}^{\mathrm{T}} \mathbf{x}_{n, i} ; \boldsymbol{\delta}, \lambda_{0}\right)\right] \mathbf{z}_{n, i} \mathbf{x}_{n, i}^{\mathrm{T}} \\
& +\frac{1}{n} \sum_{i=1}^{n}\left[\tilde{g}^{\prime}\left(\boldsymbol{\delta}^{\mathrm{T}} \mathbf{x}_{n, i} ; \boldsymbol{\delta}, \lambda_{0}\right)-g^{\prime}\left(\boldsymbol{\delta}_{0}^{\mathrm{T}} \mathbf{x}_{n, i}\right)\right] \mathbf{z}_{n, i} \mathbf{x}_{n, i}^{\mathrm{T}} \\
& +\frac{1}{n} \sum_{i=1}^{n} g^{\prime}\left(\boldsymbol{\delta}_{0}^{\mathrm{T}} \mathbf{x}_{n, i}\right) \mathbf{z}_{n, i} \mathbf{x}_{n, i}^{\mathrm{T}} \\
= & \frac{1}{n} \sum_{i=1}^{n} g^{\prime}\left(\boldsymbol{\delta}_{0}^{\mathrm{T}} \mathbf{x}_{n, i}\right) \mathbf{z}_{n, i} \mathbf{x}_{n, i}^{\mathrm{T}}+o_{p}(1) .
\end{aligned}
$$

when $n h^{2} \rightarrow \infty$.

Next, it follows by straightforward calculation, Lemma 3 and Lemma 4 that

$$
-\frac{1}{n} \frac{\partial \mathbf{m}_{n}(\boldsymbol{\delta}, \lambda)}{\partial \lambda}=\frac{1}{n} Z_{n}^{\mathrm{T}}\left[I_{n}-S_{n h}\left(\boldsymbol{\delta}_{0}^{\mathrm{T}} X_{n} ; \boldsymbol{\delta}_{0}\right)\right] M_{n} \mathbf{g}\left(\boldsymbol{\delta}_{0}^{\mathrm{T}} X_{n}\right)+o_{P}(1),
$$

and

$$
\frac{1}{\sqrt{n}} \widetilde{\mathbf{m}}_{n}\left(\boldsymbol{\theta}_{0}\right)=\frac{1}{\sqrt{n}} Z_{n}^{\mathrm{T}}\left[I_{n}-S_{n h}\left(\boldsymbol{\delta}_{0}^{\mathrm{T}} X_{n} ; \boldsymbol{\delta}_{0}\right)\right] \boldsymbol{\epsilon}_{n}+O\left(n^{1 / 2} h^{2}\right) .
$$

Using results (A.3) - (A.6), $J(\boldsymbol{\alpha})-J\left(\boldsymbol{\alpha}_{0}\right)=O\left(n^{-1 / 2}\right)$, Condition (8), Lemma 4 and $n h^{4} \rightarrow 0$, we obtain that

$$
\left[-\frac{1}{n} \frac{\partial \widetilde{\mathbf{m}}_{n}(\boldsymbol{\theta})}{\partial \boldsymbol{\theta}^{\mathrm{T}}}\right]^{\mathrm{T}} A_{n} \frac{1}{\sqrt{n}} \widetilde{\mathbf{m}}_{n}\left(\boldsymbol{\theta}_{0}\right)=O_{P}(1)
$$


and

$$
\left[-\frac{1}{n} \frac{\partial \widetilde{\mathbf{m}}_{n}(\boldsymbol{\theta})}{\partial \boldsymbol{\theta}^{\mathrm{T}}}\right]^{\mathrm{T}} A_{n}\left[-\frac{1}{n} \frac{\partial \widetilde{\mathbf{m}}_{n}(\overline{\boldsymbol{\theta}})}{\partial \boldsymbol{\theta}^{\mathrm{T}}}\right]=\Gamma^{\mathrm{T}} A \Gamma+o_{P}(1) .
$$

Hence, for any $\varepsilon>0$, there exists some constant $B_{2}>0$ such that when $n$ is sufficiently large, we have

$$
P\left(R_{n 1} \leq \sqrt{n} B_{2}\left\|\boldsymbol{\theta}-\boldsymbol{\theta}_{0}\right\| \text { for all } \boldsymbol{\theta} \in \mathcal{B}_{n}\right) \geq 1-\varepsilon / 2
$$

where $R_{n 1}=\left(\boldsymbol{\theta}-\boldsymbol{\theta}_{0}\right)^{\mathrm{T}}\left[-\frac{1}{n} \frac{\partial \widetilde{\mathbf{m}}_{n}(\boldsymbol{\theta})}{\partial \boldsymbol{\theta}^{\mathrm{T}}}\right]^{\mathrm{T}} A_{n} \widetilde{\mathbf{m}}_{n}\left(\boldsymbol{\theta}_{0}\right)$.

Moreover, since $\Gamma^{\mathrm{T}} A \Gamma$ is a positive definite matrix, its minimum eigenvalue is larger than zero, which leads to $\Gamma^{\mathrm{T}} A \Gamma \geq C$. This implies that for the above given $\varepsilon$, there exists some constant $B_{3}>0$ such that for sufficiently large $n$,

$$
P\left(R_{n 2} \geq n B_{3}\left\|\boldsymbol{\theta}-\boldsymbol{\theta}_{0}\right\|^{2} \text { for all } \boldsymbol{\theta} \in \mathcal{B}_{n}\right) \geq 1-\varepsilon / 2
$$

where $R_{n 2}=\left(\boldsymbol{\theta}-\boldsymbol{\theta}_{0}\right)^{\mathrm{T}}\left[-\frac{1}{n} \frac{\partial \widetilde{\mathbf{m}}_{n}(\boldsymbol{\theta})}{\partial \boldsymbol{\theta}^{\mathrm{T}}}\right]^{\mathrm{T}} A_{n}\left[-\frac{\partial \widetilde{\mathbf{m}}_{n}(\overline{\boldsymbol{\theta}})}{\partial \boldsymbol{\theta}^{\mathrm{T}}}\right]\left(\boldsymbol{\theta}-\boldsymbol{\theta}_{0}\right)$.

Let $B=2 B_{2} / B_{3}$, we have by (A.2), (A.8) and (A.9) that

$$
\begin{aligned}
& P\left(\left(\boldsymbol{\theta}-\boldsymbol{\theta}_{0}\right)^{\mathrm{T}} \frac{\partial \widetilde{Q}_{n}(\boldsymbol{\theta})}{\partial \boldsymbol{\theta}}>0 \text { for all } \boldsymbol{\theta} \in \mathcal{B}_{n}\right) \\
\geq & P\left(R_{n 1}-R_{n 2} \leq-B B_{2} \text { for all } \boldsymbol{\theta} \in \mathcal{B}_{n}\right) \geq 1-\varepsilon .
\end{aligned}
$$

Next, we will establish the asymptotic distribution of $\hat{\boldsymbol{\theta}}$. Since $\hat{\boldsymbol{\theta}}-\boldsymbol{\theta}_{0}=O_{P}\left(n^{-1 / 2}\right)$, it follows by $\frac{\partial \tilde{Q}_{n}(\hat{\boldsymbol{\theta}})}{\partial \boldsymbol{\theta}}=\mathbf{0},\left(\right.$ A.2), (A.6), (A.7) and $J(\hat{\boldsymbol{\alpha}})-J\left(\boldsymbol{\alpha}_{0}\right)=o_{P}(1)$ that

$$
\sqrt{n}\left(\hat{\boldsymbol{\theta}}-\boldsymbol{\theta}_{0}\right)=\left(\Gamma^{\mathrm{T}} A \Gamma\right)^{-1} \Gamma^{\mathrm{T}} A \frac{1}{\sqrt{n}} Z_{n}^{\mathrm{T}}\left[I_{n}-S_{n h}\left(\boldsymbol{\delta}_{0}^{\mathrm{T}} X_{n} ; \boldsymbol{\delta}_{0}\right)\right] \boldsymbol{\epsilon}_{n}+o_{P}(1),
$$

where $\tilde{\boldsymbol{\theta}}$ lies between $\hat{\boldsymbol{\theta}}$ and $\boldsymbol{\theta}_{0}$, hence $\tilde{\boldsymbol{\theta}}-\boldsymbol{\theta}_{0}=O_{P}\left(n^{-1 / 2}\right)$.

Further, it follows from Lemma 4 that

$$
\frac{1}{\sqrt{n}} Z_{n}^{\mathrm{T}}\left[I_{n}-S_{n h}\left(\boldsymbol{\delta}_{0}^{\mathrm{T}} X_{n} ; \boldsymbol{\delta}_{0}\right)\right] \boldsymbol{\epsilon}_{n} \stackrel{D}{\rightarrow} \mathbf{N}\left(\mathbf{0}, \sigma^{2} \Sigma\right) .
$$

Hence, we obtain that

$$
\sqrt{n}\left(\hat{\boldsymbol{\theta}}-\boldsymbol{\theta}_{0}\right) \stackrel{D}{\rightarrow} \mathbf{N}\left(\mathbf{0}, \sigma^{2}\left(\Gamma^{\mathrm{T}} A \Gamma\right)^{-1} \Gamma^{\mathrm{T}} A \Sigma A^{\mathrm{T}} \Gamma\left(\Gamma^{\mathrm{T}} A \Gamma\right)^{-1}\right) .
$$

Now we consider the asymptotic distribution of the original parameters estimator $\hat{\boldsymbol{\xi}}=\left(\hat{\boldsymbol{\delta}}^{\mathrm{T}}, \hat{\lambda}\right)^{\mathrm{T}}$. Since we have by (A.1) that

$$
\sqrt{n}\left(\hat{\boldsymbol{\xi}}-\boldsymbol{\xi}_{0}\right)=J_{1} \sqrt{n}\left(\hat{\boldsymbol{\theta}}-\boldsymbol{\theta}_{0}\right)+o_{P}(1)
$$


the asymptotic normality of $\sqrt{n}\left(\hat{\boldsymbol{\xi}}-\boldsymbol{\xi}_{0}\right)$ follows.

Proof of Theorem 2: The optimal weighting matrix for $A_{n}$ is $\Sigma_{n}^{-1}$ by generalized Schwartz inequality. To proof theorem 2, it only needs to prove that $\hat{\Sigma}_{n}-\Sigma_{n}=$ $o_{P}(1)$, and then using the same lines as establishing Theorem 1 with $A_{n}$ replaced by $\Sigma_{n}^{-1}+o_{P}(1)$.

Since for $a=0,1$, we have by straightforward calculation, Taylor expansion and Condition (2) that

$$
\begin{aligned}
& \sup _{\mathbf{x}} \frac{1}{n} \sum_{j=1}^{n}\left|\frac{K_{h}\left(\boldsymbol{\delta}^{\mathrm{T}}\left(\mathbf{x}_{n, j}-\mathbf{x}\right)\right)}{f\left(\boldsymbol{\delta}^{\mathrm{T}} \mathbf{x}\right)}-\frac{K_{h}\left(\hat{\boldsymbol{\delta}}^{\mathrm{T}}\left(\mathbf{x}_{n, j}-\mathbf{x}\right)\right)}{f\left(\hat{\boldsymbol{\delta}}^{\mathrm{T}} \mathbf{x}\right)}\right| \cdot\left\|\mathbf{z}_{n, j}\right\|^{a} \\
\leq & \sup _{\mathbf{x}} \frac{1}{n} \sum_{j=1}^{n}\left|K_{h}\left(\boldsymbol{\delta}^{\mathrm{T}}\left(\mathbf{x}_{n, j}-\mathbf{x}\right)\right)\left[1 / f\left(\boldsymbol{\delta}^{\mathrm{T}} \mathbf{x}\right)-1 / f\left(\hat{\boldsymbol{\delta}}^{\mathrm{T}} \mathbf{x}\right)\right]\right| \cdot\left\|\mathbf{z}_{n, j}\right\|^{a} \\
& \quad+\sup _{\mathbf{x}} \frac{1}{n} \sum_{j=1}^{n}\left|\left[K_{h}\left(\boldsymbol{\delta}^{\mathrm{T}}\left(\mathbf{x}_{n, j}-\mathbf{x}\right)\right)-K_{h}\left(\hat{\boldsymbol{\delta}}^{\mathrm{T}}\left(\mathbf{x}_{n, j}-\mathbf{x}\right)\right)\right] / f\left(\hat{\boldsymbol{\delta}}^{\mathrm{T}} \mathbf{x}\right)\right| \cdot\left\|\mathbf{z}_{n, j}\right\|^{a} \\
= & O_{P}\left(n^{-1 / 2} h^{-1}\right)=o_{P}(1) .
\end{aligned}
$$

Next, it follows by Lemma 2(1) and some calculation that

$$
\mathbf{s}_{n h}\left(\boldsymbol{\delta}^{\mathrm{T}} \mathbf{x} ; \boldsymbol{\delta}\right)=\frac{1}{n f\left(\boldsymbol{\delta}^{\mathrm{T}} \mathbf{x}\right)}\left(K_{h}\left(\boldsymbol{\delta}^{\mathrm{T}}\left(\mathbf{x}_{n, 1}-\mathbf{x}\right)\right), \cdots, K_{h}\left(\boldsymbol{\delta}^{\mathrm{T}}\left(\mathbf{x}_{n, n}-\mathbf{x}\right)\right)\right)\{1+o(1)\} .
$$

Then we obtain by tedious calculation and Condition (2) that

$$
\frac{1}{n} Z_{n}^{\mathrm{T}}\left[S_{n h}\left(\boldsymbol{\delta}_{0}^{\mathrm{T}} X_{n} ; \boldsymbol{\delta}_{0}\right)-S_{n h}\left(\hat{\boldsymbol{\delta}}^{\mathrm{T}} X_{n} ; \hat{\boldsymbol{\delta}}\right)\right]\left[I_{n}-S_{n h}\left(\boldsymbol{\delta}_{0}^{\mathrm{T}} X_{n} ; \boldsymbol{\delta}_{0}\right)\right]^{\mathrm{T}} Z_{n}=o_{P}(1),
$$

and

$$
\frac{1}{n} Z_{n}^{\mathrm{T}}\left[S_{n h}\left(\boldsymbol{\delta}_{0}^{\mathrm{T}} X_{n} ; \boldsymbol{\delta}_{0}\right)-S_{n h}\left(\hat{\boldsymbol{\delta}}^{\mathrm{T}} X_{n} ; \hat{\boldsymbol{\delta}}\right)\right]\left[S_{n h}\left(\boldsymbol{\delta}_{0}^{\mathrm{T}} X_{n} ; \boldsymbol{\delta}_{0}\right)-S_{n h}\left(\hat{\boldsymbol{\delta}}^{\mathrm{T}} X_{n} ; \hat{\boldsymbol{\delta}}\right)\right]^{\mathrm{T}} Z_{n}=o_{P}(1) .
$$

Combining the above results, we obtain that $\hat{\Sigma}_{n}-\Sigma_{n}=o_{P}(1)$.

Proof of Theorem 3: Let $v=\boldsymbol{\delta}_{0}^{\mathrm{T}} \mathbf{x}, v_{n, i}=\boldsymbol{\delta}_{0}^{\mathrm{T}} \mathbf{x}_{n, i}, \hat{v}=\hat{\boldsymbol{\delta}}^{\mathrm{T}} \mathbf{x}$, and $\hat{v}_{n, i}=\hat{\boldsymbol{\delta}}^{\mathrm{T}} \mathbf{x}_{n, i}$. It then follows by straightforward calculation that

$$
\hat{g}(\hat{v} ; \hat{\boldsymbol{\delta}}, \hat{\lambda})-g(v)=\hat{g}^{\prime}\left(v ; \boldsymbol{\delta}_{0}, \hat{\lambda}\right)(\hat{v}-v)\left\{1+o_{P}(1)\right\}+\hat{g}\left(v ; \boldsymbol{\delta}_{0}, \hat{\lambda}\right)-g(v) .
$$


It can be seen by Lemma 2(3) (5) with $h$ being replaced by $h_{1}$ that

$$
\begin{aligned}
\hat{g}^{\prime}\left(v ; \boldsymbol{\delta}_{0}, \hat{\lambda}\right)-g^{\prime}(v)= & h_{1}^{-1}\left(\lambda_{0}-\hat{\lambda}\right) \mathbf{s}_{n h_{1}}^{*}\left(v ; \boldsymbol{\delta}_{0}\right) W_{n} Y_{n}+h_{1}^{-1} \mathbf{s}_{n h_{1}}^{*}\left(v ; \boldsymbol{\delta}_{0}\right) \boldsymbol{\epsilon}_{n} \\
& +\left[\hat{g}^{\prime}\left(v ; \boldsymbol{\delta}_{0}, \lambda_{0}\right)-g^{\prime}(v)-h_{1}^{-1} \mathbf{s}_{n h_{1}}^{*}\left(v ; \boldsymbol{\delta}_{0}\right) \boldsymbol{\epsilon}_{n}\right] \\
= & O_{P}\left(\left\{n h_{1}^{2}\right\}^{-1 / 2}+h_{1}\right)+h_{1}^{-1} \mathbf{s}_{n h_{1}}^{*}\left(v ; \boldsymbol{\delta}_{0}\right) \boldsymbol{\epsilon}_{n} .
\end{aligned}
$$

Hence, it follows by Theorem 1 and Chebyshev inequality that

$$
\sqrt{n h_{1}} \hat{g}^{\prime}\left(v ; \boldsymbol{\delta}_{0}, \hat{\lambda}\right)(\hat{v}-v)=h_{1}^{-1 / 2} \mathbf{s}_{n h_{1}}^{*}\left(v ; \boldsymbol{\delta}_{0}\right) \boldsymbol{\epsilon}_{n}+o_{P}(1)=o_{P}(1)
$$

using $n h_{1}^{2} \rightarrow \infty$.

Moreover, we can seen by Lemma 2(2) (3) and $\hat{\lambda}-\lambda_{0}=O_{P}\left(n^{-1 / 2}\right)$ that

$$
\begin{aligned}
& \sqrt{n h_{1}}\left\{\hat{g}\left(v ; \boldsymbol{\delta}_{0}, \hat{\lambda}\right)-g(v)\right\} \\
= & (1,0)\left\{n^{-1} \Psi_{n h_{1}}\left(v ; \boldsymbol{\delta}_{0}\right)\right\}^{-1} \sqrt{n^{-1} h_{1}} \mathcal{X}_{n h_{1}}^{\mathrm{T}}\left(v ; \boldsymbol{\delta}_{0}\right) K_{n h_{1}}\left(v ; \boldsymbol{\delta}_{0}\right) \boldsymbol{\epsilon}_{n} \\
& +O_{P}\left(h_{1}^{1 / 2}\right)+\sqrt{n h_{1}} \frac{1}{2} \mu_{2} h_{1}^{2} g^{\prime \prime}(v)+o_{P}\left(\left\{n h_{1}^{5}\right\}^{1 / 2}\right)
\end{aligned}
$$

Next, it follows by straightforward calculation that

$$
\sqrt{n^{-1} h_{1}} E\left(\mathcal{X}_{n h_{1}}^{\mathrm{T}}\left(v ; \boldsymbol{\delta}_{0}\right) \mathcal{K}_{n h_{1}}\left(v ; \boldsymbol{\delta}_{0}\right) \boldsymbol{\epsilon}_{n}\right)=\mathbf{0}
$$

and

$$
n^{-1} h_{1} \operatorname{cov}\left(\mathcal{X}_{n h_{1}}^{\mathrm{T}}\left(v ; \boldsymbol{\delta}_{0}\right) \mathcal{K}_{n h_{1}}\left(v ; \boldsymbol{\delta}_{0}\right) \boldsymbol{\epsilon}_{n}\right)=\sigma^{2} f(v)\left(\begin{array}{cc}
\nu_{0} & 0 \\
0 & \nu_{2}
\end{array}\right)+o(1) .
$$

Furthermore, for any nonzero constants $b_{1}, b_{2}$ and $\varepsilon>0$, we obtain that

$$
\left\{\sqrt{n^{-1} h_{1}}\right\}^{2+\varepsilon} \sum_{i=1}^{n} E\left|\left(b_{1}+b_{2} \frac{v_{n, i}-v}{h_{1}}\right) K_{n h_{1}}\left(v_{n, i}-v\right) \epsilon_{n, i}\right|^{2+\varepsilon}=O\left(\left\{n h_{1}\right\}^{-\frac{\varepsilon}{2}}\right)
$$

by Cr inequality and Condition (2). Hence it follows by Central Limit Theorem that

$$
\sqrt{n^{-1} h_{1}} \mathcal{X}_{n h_{1}}^{\mathrm{T}}\left(v ; \boldsymbol{\delta}_{0}\right) \mathcal{K}_{n h_{1}}\left(v ; \boldsymbol{\delta}_{0}\right) \boldsymbol{\epsilon}_{n} \stackrel{D}{\rightarrow} \mathbf{N}\left(\mathbf{0}, \sigma^{2} f(v) \operatorname{diag}\left(\nu_{0}, \nu_{2}\right)\right) .
$$

Then Theorem 2 follows from (A.10) - (A.13), $\hat{\boldsymbol{\delta}}-\boldsymbol{\delta}_{0}=O_{P}\left(n^{-1 / 2}\right)$ and Lemma 2(1).

Proof of Theorem 4: Let $v=\boldsymbol{\delta}_{0}^{\mathrm{T}} \mathbf{x}, v_{n, i}=\boldsymbol{\delta}_{0}^{\mathrm{T}} \mathbf{x}_{n, i}, \hat{v}=\hat{\boldsymbol{\delta}}^{\mathrm{T}} \mathbf{x}$, and $\hat{v}_{n, i}=\hat{\boldsymbol{\delta}}^{\mathrm{T}} \mathbf{x}_{n, i}$. It can be seen from the proof of Theorem 3 that

$$
\hat{g}(\hat{v} ; \hat{\boldsymbol{\delta}}, \hat{\lambda})-g(v)-\operatorname{bias}\{\hat{g}(\hat{v} ; \hat{\boldsymbol{\delta}}, \hat{\lambda})\}=\mathbf{s}_{n h_{1}}\left(v ; \boldsymbol{\delta}_{0}\right) \boldsymbol{\epsilon}_{n}+O_{P}\left(n^{-1 / 2}\right)
$$


uniformly in $v \in[a, b]$. Next, we will prove that

$$
\sup _{v \in[a, b]}\left|\mathbf{s}_{n h_{1}}\left(v ; \boldsymbol{\delta}_{0}\right) \boldsymbol{\epsilon}_{n}-\frac{1}{n f(v)} \sum_{i=1}^{n} K_{h_{1}}\left(v_{n, i}-v\right) \epsilon_{n, i}\right|=o_{P}\left(\left\{n h_{1} \log n\right\}^{-1 / 2}\right) .
$$

It follows from the proof of Lemma 2(1) and (3.3) that

$$
n\left\{\Psi_{n h_{1}}\left(v ; \boldsymbol{\delta}_{0}\right)\right\}^{-1}=\frac{1}{f(v)}\left(\begin{array}{cc}
1 & 0 \\
0 & 1 / \mu_{2}
\end{array}\right)+o(1 / \log n)
$$

uniformly in $v \in[a, b]$. This, together with Lemma 1 leads to (A.14). Hence, to prove the theorem, it suffices to show by Lemma 1, (3.3) and the proof of Theorem 3 that

$$
\begin{gathered}
P\left(\left(-2 \log \left\{h_{1} /(b-a)\right\}\right)^{1 / 2} \sup _{v \in[a, b]}\left|\left\{n h_{1} f(v) \sigma^{2} \nu_{0}\right\}^{-1 / 2} \sum_{i=1}^{n} K\left(\frac{v_{n, i}-v}{h_{1}}\right) \epsilon_{n, i}\right|-l_{n} \leq x\right) \\
\rightarrow \exp (-2 \exp (-x)) .
\end{gathered}
$$

Let $L_{1}(v)=\sum_{i=1}^{n} K\left(\frac{v_{n, i}-v}{h_{1}}\right) \epsilon_{n, i}$, and divide the interval $[a, b]$ into $n$ subintervals $J_{1}=\left[a, d_{1}\right], J_{r}=\left(d_{r-1}, d_{r}\right], r=2, \cdots, n$, where $d_{r}=a+\frac{b-a}{n} r$. Define $\tilde{v}_{n, i}=$ $d_{r} I\left(v_{n, i} \in J_{r}\right), r=1, \cdots, n$. It is obvious that $\left|v_{n, i}-\tilde{v}_{n, i}\right|=O\left(n^{-1}\right)$. Then it follows by Chebyshev inequality and conditions of $K(\cdot)$ that

$$
\begin{aligned}
L_{1}(v) & =\sum_{i=1}^{n}\left[K\left(\frac{v_{n, i}-v}{h_{1}}\right)-K\left(\frac{\tilde{v}_{n, i}-v}{h_{1}}\right)\right] \epsilon_{n, i}+\sum_{i=1}^{n} K\left(\frac{\tilde{v}_{n, i}-v}{h_{1}}\right) \epsilon_{n, i} \\
& =o_{P}(1)+\sum_{r=1}^{n} K\left(\frac{d_{r}-v}{h_{1}}\right) \sum_{i=1}^{n} I\left(v_{n, i} \in J_{r}\right) \epsilon_{n, i} \equiv o_{P}(1)+L_{2}(v) .
\end{aligned}
$$

Define $\zeta_{t}=\sum_{r=1}^{t} \sum_{i=1}^{n} I\left(v_{n, i} \in J_{r}\right) \epsilon_{n, i}=\sum_{i=1}^{n} I\left(a \leq v_{n, i} \leq d_{t}\right) \epsilon_{n, i}$ and $\zeta_{0}=0$. If $v_{n, i}$ can be seen as a sequence of iid random variables, then $\zeta_{t}$ is the hybrids of empirical and partial sums processes. It follows by Theorem 2.1 of Horvath (2000) that

$$
\sup _{t}\left|\zeta_{t}-n^{1 / 2} \mathcal{W}\left(G\left(d_{t}\right)\right)\right|=O_{P}\left(n^{1 / 4}\right)
$$

where $\mathcal{W}(\cdot)$ is a Wiener process and $G(s)=\sigma^{2} \int_{a}^{s} f(t) d t$.

Since it follows by (A.16) and conditions of $K(\cdot)$ that

$$
\begin{aligned}
& \sup _{v \in[a, b]}\left|\sum_{r=1}^{n-1}\left[K\left(\frac{d_{r+1}-v}{h_{1}}\right)-K\left(\frac{d_{r}-v}{h_{1}}\right)\right]\left[\zeta_{r}-n^{1 / 2} \mathcal{W}\left(G\left(d_{r}\right)\right)\right]\right| \\
\leq & \max _{r}\left|\zeta_{r}-n^{1 / 2} \mathcal{W}_{n}\left(G\left(d_{r}\right)\right)\right| \sup _{v \in[a, b]} \sum_{r=1}^{n-1}\left|K\left(\frac{d_{r+1}-v}{h_{1}}\right)-K\left(\frac{d_{r}-v}{h_{1}}\right)\right|=O_{P}\left(n^{1 / 4}\right),
\end{aligned}
$$


we have

$$
L_{2}(v)=n^{1 / 2} \int_{a}^{b} K\left(\frac{s-v}{h_{1}}\right) d \mathcal{W}(G(s))+O_{P}\left(\{\log n\}^{1 / 2}+n^{1 / 4}\right) .
$$

by the bounded variation of $K(\cdot)$ and the following property of Wiener process (see Page 44 in Csorgo and Revesz, 1981)

$$
\sup _{s \in[a, b]}|\mathcal{W}(G(s+\eta))-\mathcal{W}(G(s))|=O\left(\{\eta \log (1 / \eta)\}^{1 / 2}\right) \quad \text { a.s. },
$$

where $\eta$ is any small number.

Combining the above results, we can obtain that

$$
\sup _{v \in[a, b]}\left|\left(n h_{1}\right)^{-1 / 2} L_{1}(v)-h_{1}^{-1 / 2} \int_{a}^{b} K\left(\frac{s-v}{h_{1}}\right) d \mathcal{W}(G(s))\right|=O_{P}\left(\left\{n h_{1}^{2}\right\}^{-1 / 4}+\left\{\frac{\log n}{n h_{1}}\right\}^{1 / 2}\right) .
$$

Following the similar proofs of lemmas in Härdle (1989), we have

$$
\sup _{v \in[a, b]}\left|h_{1}^{-1 / 2} \int_{a}^{b} K\left(\frac{s-v}{h_{1}}\right) d \mathcal{W}(G(s))-L_{3}(v)\right|=O_{P}\left(h_{1}^{1 / 2}\right),
$$

where $L_{3}(v)=h_{1}^{-1 / 2} \int_{a}^{b} K\left(\frac{s-v}{h_{1}}\right)\left[\sigma^{2} f(s)\right]^{1 / 2} d \mathcal{W}(s-a)$, and

$$
\sup _{v \in[a, b]}\left|\left[\sigma^{2} f(v)\right]^{-1 / 2} L_{3}(v)-h_{1}^{-1 / 2} \int_{a}^{b} K\left(\frac{s-v}{h_{1}}\right) d \mathcal{W}(s-a)\right|=O_{P}\left(h_{1}^{1 / 2}\right) .
$$

Then (A.15) follows by (A.17)-(A.19), arguments' transformation, Theorem 2 and Theorem 3.1 of Bickel and Rosenblatt (1973).

\section{Acknowledgments}

The author thanks the editor Zhenlin Yang and two referees for their valuable comments and suggestions. The article is supported by National Science Foundation of China (Grant 11271242), Shuguang Program of Shanghai Education Development Foundation and Shanghai Municipal Education Commission (2015), and Key Laboratory of Mathematical Economics (SUFE), Ministry of Education of China.

\section{References}

Basile, R., Gress, B., 2005. Semiparametric spatial auto-covariance models of regional growth behavior in Europe. Region et Developpement 21, 97-118. 
Basile, R., Durban, M., Minguez, R., Montero, J. M., Mur, J., 2014. Modeling regional economic dynamics: spatial dependence, spatial heterogenity and nonlinearities. Journal of Economic Dynamics and Control 48, 229-245.

Bellman, R. E., 1961. Adaptive control processes. Princeton, NJ: Princeton University Press.

Bickel, P. L., Rosenblatt, M., 1973. On some global measures of the derivations of density function estimates. The Annals of Statistics 1, 1071-1095.

Carroll, R. J., Fan, J., Gijbels, I., Wand, M. P., 1997. Generalized partially linear single-index models. Journal of the American Statistical Association 92, 477489.

Csorgo, M., Revesz, P., 1981. Strong approximations in probability and statistics. Academic Press, New York.

Fan, J., Gijbels, I., 1996. Local polynomial modelling and its applications. Chapman and Hall.

Fan, J., Zhang, W., 2000. Simultaneous confidence bands and hypothesis testing in varying-coefficient models. Scandinavian Journal of Statistics 27, 715-731.

Härdle, W., 1989. Asymptotic maximal deviation of M-smoothers. Journal of Multivariate Analysis 29, 163-179.

Härdle, W., 1994. Applied nonparametric regression. Cambridge.

Horn, R., Johnson, C., 1985. Matrix Analysis. New York: Cambridge University Press.

Horvath, L., 2000. Approximations for hybrids of empirical and partial sums processes. Journal of Statistical Planning and Inference 88, 1-18.

Ichimura, H., 1993. Semiparametric least squares (SLS) and weighted SLS estimation of single-index models. Journal of Econometrics 58, 71-120.

Kelejian, H. H., Prucha, I. R., 1998. A generalized spatial two-stage least squares procedure for estimating a spatial autoregressive model with autoregressive disturbance. Journal of Real Estate Finance and Economics 17, 99-121.

Kelejian, H. H., Prucha, I. R., 1999. A generalized moments estimator for the autoregressive parameter in a spatial model. International Economic Review 40, 509-533.

Lee, L., 2004. Asymptotic distributions of quasi-maximum likelihood estimators for spatial autoregressive models. Econometrica 72, 1899C1925.

Lee, L., 2007. GMM and 2SLS estimation of mixed regressive spatial autoregressive models. Journal of Econometrics 137, 489-514. 
LeSage, J., Pace, K., 2009. Introduction to spatial econometrics. CRC Press, Boca Raton.

Liang, H., Liu, X., Li, R., Tsai, C., 2010. Estimation and testing for paritally linear single-index models. Annals of Statistics 38, 3811-3836.

Linton, O., 1995. Second order approximation in the partially linear regression model. Econometrica 63, 1079-1112.

Mack, Y. P., Silverman, B. W., 1982. Weak and strong uniform consistency of kernel regression estimates. Zeitschrift fur Wahrscheinlichkeitstheorie und verwandte Gebiete $61,405-415$.

Ortega, J. M., Rheinboldt, W. C., 1973. Iterative solution of nonlinear equations in several variables. New York: Academic.

Pace, P. K., Barry, R., Slawson, Jr., V. C., Sirmans, C. F., 2004. Simultaneous spatial and functional form transformation. In: Anselin, L., Florax, R., Rey, S. J. (Eds), Advances in Spatial Econometrics. Springer-Verlag, Berlin, 194-224.

Paelinck, J. H. P., Klaassen, L. H., 1979. Spatial Econometrics. Gower Press, Aldershot.

Powell, J. L., Stock, J. H., Stoker, T. M., 1989. Semiparametric estimation of single coefficients. Econometrica 57, 1403-1430.

$\mathrm{Su}$, L., 2012. Semiparametric GMM estimation of spatial autoregressive models. Journal of Econometrics 167, 543-560.

Su, L., Jin, S., 2010. Profile quasi-maximum likelihood estimation of partially linear spatial autoregressive models. Journal of Econometrics 157, 18-33.

Sun, Y., Yan, H., Zhang, W., Lu, Z., 2014. A semiparametric spatial dynamic model. The Annals of Statistics 42, 700-727.

Wang, J., Xue, L., Zhu, L., Chong, Y., 2010. Estimation for a partial-linear singleindex model. The Annals of Statistics 38, 246-274.

Xia, Y., 2006. Asymptotic distributions for two estimators of the single-index model. Econometric Theory 22, 1112-1137.

Yang, Z., Li, C., Tse, Y. K., 2006. Functional form and spatial dependence in dynamic panels. Economics Letters 91, 138-145.

Yu, Y., Ruppert, D., 2002. Penalized spline estimation for partially linear singleindex models. Journal of the American Statistical Association 97, 1042-1054. 